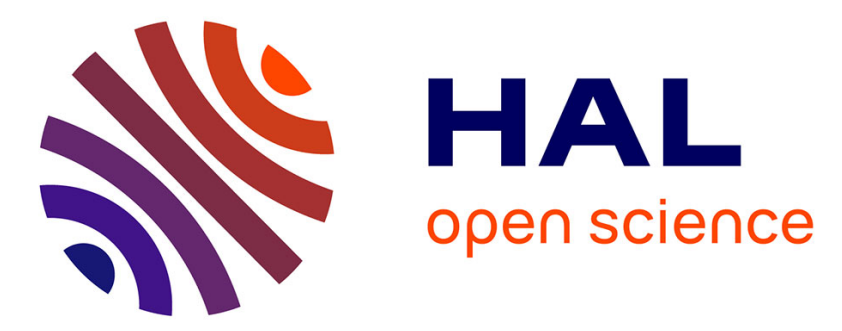

\title{
Fast and accurate MAS-DNP simulations of large spin ensembles
}

\author{
Frederic Mentink-Vigier, Shimon Vega, Gaël de Paëpe
}

\section{To cite this version:}

Frederic Mentink-Vigier, Shimon Vega, Gaël de Paëpe. Fast and accurate MAS-DNP simulations of large spin ensembles. Physical Chemistry Chemical Physics, 2017, 19 (5), pp.3506-3522. 10.1039/c6cp07881h . hal-02048457

\section{HAL Id: hal-02048457 https://hal.science/hal-02048457}

Submitted on 29 Sep 2021

HAL is a multi-disciplinary open access archive for the deposit and dissemination of scientific research documents, whether they are published or not. The documents may come from teaching and research institutions in France or abroad, or from public or private research centers.
L'archive ouverte pluridisciplinaire HAL, est destinée au dépôt et à la diffusion de documents scientifiques de niveau recherche, publiés ou non, émanant des établissements d'enseignement et de recherche français ou étrangers, des laboratoires publics ou privés. 


\title{
Fast and accurate MAS-DNP simulations of large spin ensembles
}

\author{
Frédéric Mentink-Vigier,ab Shimon Vega,c Gaël De Paëpe*ab \\ a Univ. Grenoble Alpes, INAC, MEM, F-38000 Grenoble, France. \\ E-mail: gael.depaepe@cea.fr \\ b CEA, INAC, MEM, F-38000 Grenoble, France and \\ c Weizmann institute of Science, Rehovot, Israel
}

(Dated:)

\begin{abstract}
A deeper understanding of parameters affecting Magic Angle Spinning Dynamic Nuclear Polarization (MAS-DNP), an emerging nuclear magnetic resonance hyperpolarization method, is crucial for the development of new polarizing agents and the successful implementation of the technique at higher magnetic fields $(>10 \mathrm{~T})$. Such progress is currently impeded by computational limitation which prevents the simulation of large spin ensembles (electron as well as nuclear spins) and to accurately describe the interplay between all the multiple key parameters at play.

In this work, we present an alternative approach to existing Cross-Effect and Solid-Effect MASDNP codes that yields fast and accurate simulations. More specifically we describe the model, the associated Liouville-based formalism (Bloch-type derivation and/or Landau-Zener approximations) and the linear time algorithm that allows computing MAS-DNP mechanisms with unprecedented time savings. As a result, one can easily scan through multiple parameters and disentangle their mutual influences. In addition, the simulation code is able to handle multiple electrons and protons, which allows probing the effect of (hyper)polarizing agents concentration, as well as fully revealing the interplay between the polarizing agent structure and the hyperfine couplings, nuclear dipolar couplings, nuclear relaxation times, both in terms of depolarization effect, but also of polarization gain and buildup times.
\end{abstract}

The combination of Dynamic Nuclear Polarization (DNP) and Magic Angle Spinning (MAS) is currently deeply impacting high-field solid-state Nuclear Magnetic Resonance (NMR). Although DNP is not a recent phenomenon, $[1,2]$ the potential of the technique for high magnetic fields was only recently demonstrated thanks to the pioneering work carried out in the Griffin laboratory [3-6]. Following this effort and the introduction of commercial systems [7], DNP experiments are currently carried out in an increasing number of laboratories, continuously expanding the scope of the technique.[8-13] The interest stems from the huge gain in NMR sensitivity obtainable though DNP, where the large intrinsic polarization of electrons is transferred to nuclei, using appropriate microwave $(\mu \mathrm{w})$ irradiation. So far, most DNP experiments are conducted successfully at 10-20 $\mathrm{T}$ at around $100 \mathrm{~K}$ using high power continuous wave $(\mathrm{CW}) \mu \mathrm{w}$ sources often combined with the use of binitroxide radicals as sources of free electrons, as introduced in the Griffin group $[3,14,15]$. Current on-going effort include improved polarizing agents and optimized sample preparation protocols [16-33], while challenging instrumental developments are also under progress in several laboratories, such as the design of more flexible high power $\mu \mathrm{w}$ sources, probe-heads with smaller diameter sample holders (rotors) and optimized $\mu \mathrm{w}$ coupling, the access to helium sample spinning and ultra-low temperature MAS-DNP experiments [34-42].

In addition, the field of MAS-DNP has also strongly benefited from recent improvements in theoretical understanding. Several groups have contributed from the experimental side by reporting DNP studies related to the effect of polarizing agents' concentration, sample spinning frequency, temperature, partial deuteration, electron spin relaxation times, and radical structure $[7,21,22,24,26,31,43-49]$. Despite these attempts to experimentally rationalize the performance of nitroxide biradicals, a clear picture has still not emerged, mostly because the DNP enhancement factor (defined as the ratio of signal with and without $\left.\mu \mathrm{w}, \epsilon_{\mathrm{on} / \mathrm{off}}=S_{\mathrm{on}} / S_{\mathrm{off}}\right)$ can lead to a misleading picture of the DNP performance while overestimating the true DNP gain significantly [50].

Previously, the most efficient and commonly used DNP mechanism, the Cross Effect (CE) was crudely described using arguments based on static (i.e. without sample spinning) experiments, which fails to provide valuable insights such as the dependency with respect to the magnetic field, the polarizing agent geometry, the $\mu \mathrm{w}$ power, electron/nuclei relaxation times, etc. Things have improved with the recent theoretical developments by Tycko and Thurber, as well as Vega et al. [50-54]. These contributions have not only brought valuable insights into the DNP mechanism, but also illustrated the complexity of high magnetic field experiments and proven the necessity to rely on numerical simulations to understand precisely the mechanisms at play in MAS-DNP experiments. The mechanism has been understood through numerical simulation tools able to describe a series of discrete events that occur periodically within one rotor period (rotor events) [50- 
53]. In the case of binitroxides, modeled by 2 electrons and a nucleus, four types of rotor events need to be considered:

- the $\mu \mathrm{w}$ rotor events, that induce a change in the electron polarization,

- the electron Dipolar - J (exchange) rotor events that tend to swap the electron polarization,

- the Cross-Effect rotor event (CE) that exchanges part of the electron polarization difference to the nuclear polarization of the hyperfine coupled nuclei,

- and the Solid-Effect rotor event (SE), that exchanges part of the electron polarization to the nuclear polarization of the hyperfine coupled nuclei.

The nuclear polarization under MAS-DNP conditions is typically computed through the use of advanced quantum mechanical simulations able to account for relaxation. More specifically the evolution operator was computed either in the Hilbert space by Thurber and Tycko [51] or in the Liouville space in the work of Mentink-Vigier et al. [50, 52, 54] and Mance et al. [55]. These computations are largely in agreement and were successfully used to simulate hyperpolarization as well as depolarization effects for various systems and experimental conditions, [50-55] and clearly highlight the complexity of this multi-parameter problem. The order and duration of the rotor events are directly related to the structure of the polarizing agent but also to the position and strength of the $\mu \mathrm{w}$ irradiation. For a given crystallite orientation, each rotor event type can typically occur 0,2 or 4 per rotor period. When the nuclear polarization has reached a steady-state value, the electrons and nuclei still see periodic changes in their polarization due to the rotor events and the electron and nuclear relaxation $\left(T_{1}^{e}, T_{1}^{n}\right)$. Ultimately it is important to note that the nuclear polarization can only be equal to or lower than the maximum electron polarization difference observed during the course of a rotor period at steady state $[50,54]$.

Based on these initial results, we now seek to further develop this computational approach towards its usage as a predictive tool. Challenges along this direction are numerous but offer the perspectives to perform in silico rational design of polarizing agents. This constitutes an important research direction since polarizing agent design has so far relied on empirical approach. The main hindrance in this approach is associated with the duration of MAS-DNP simulations, especially for large spin systems ( $>4$ spins) and the number of input parameters that needs to be accounted for such as the spin relaxation times, the biradical geometry, the $\mu \mathrm{w}$ power, the nuclear Larmor frequency, etc. So far full quantum calculations (Hilbert- or Liouville-based) have been limited to 3 or 4 spins because extension to larger spin system requires the introduction of approximations. For instance, size restrictions have been used and developed by Karbanov et al. in the static case $[56,57]$ while Thurber and Tycko [53] have recently reported a MAS-DNP simulation code able to compute the population changes of a $1000 \times 3$ spins system $(2$ electrons and 1 nucleus) randomly dispersed in a box using the Landau-Zener (LZ) approximation. [58] This last model allows describing the effect of biradical concentration and was used to simulate a depolarization mechanism in the absence of $\mu \mathrm{w}$ irradiation.

In this work we present another approach based on Liouville-type calculations that yields fast and accurate simulations compared to exact Liouville calculations. Since the goal is to build a tool that can possibly be used in a quasi-predictive fashion, the code needs:

- to be as fast as possible by building a periodic propagator,

- to be able to increase both the number of electron and nuclear spins while keeping a moderate sized problem (linear scaling),

- to be accurate in predicting the electron polarization difference at steady-state, i.e. account for electron $T_{1}^{e}$ and $T_{2}^{e}$ relaxation during rotor events.

The code presented is fast and flexible (as detailed below) and constitutes the first step towards predictive MAS-DNP simulations. For the 3 spins case (2 electrons and 1 nucleus), a 30-point CE field sweep profile can be computed in only 20 to 30 minutes (on a desktop computer) while keeping an excellent agreement with the previous code (based on full-Liouville calculations which take $6-8$ hours). This represents a significant improvement in time-savings, about a factor 15 for a 3 -spin system, which allows checking efficiently the effects of changing different parameters, such as the polarizing agent geometry, the external magnetic field $B_{0}$, the MAS rotation frequency, the relaxation rates and the spin-spin interaction strengths. In this model, each type of rotor event is treated separately using a simplified subspace (spanned by reduced Liouvillian operators). The relevant spin dynamics during a rotor event are computed either by relying on Bloch-type equations accounting for electronic and nuclear relaxation or using the LZ formula. The former are particularly important for rotor events that act on timescales of the order of the relaxation times, which therefore cannot be ignored. As demonstrated in the following, each rotor event is thus described with a set of first-order linear equations. The overall evolution is then computed assuming that every rotor event is independent and that the superposition principle can be applied. 
Importantly, we also show that the number of electrons and nuclei can easily be increased so as to account for multi electron effects as well as nuclear spin diffusion. This new feature can be used to compute DNP enhancement factors and polarization build-up times for the various protons present in large spin assemblies of tens to thousands of spins. This provides great insight into many key parameters, such as the electron concentration, the magnetic field dependency, polarizing agent geometries, and nuclear relaxation times of bulk versus local protons, explaining physically observed phenomena.

\section{MATERIALS AND METHODS}

The simulation codes have been written in Matlab (MATLAB and Statistics Toolbox Release 2013a, The MathWorks, Inc., Natick, Massachusetts, United States.) and optimized to minimize computational time. In particular we made use of the Suite Sparse Matlab toolbox when considering large spin system [59]. The simulations have been run on a Dell Precision T5500, using 2 Intel Xeon(R) CPU X5650 @ $2.67 \mathrm{GHz}$ (24 logical cores), using Ubuntu 15.10 as the OS.

Except when specified otherwise we performed calculations assuming a temperature of $100 \mathrm{~K}$, either on a biradical geometry close to bTbK [60] using $\left[g_{z}, g_{y}, g_{x}\right]$ $=[2.0024,2.0063,2.0097]$ for the g-tensor values, or close to TOTAPOL using $\left[g_{z}, g_{y}, g_{x}\right]=[2.0094,2.006$, $2.0017]$ as used in previous work $[52,54,61]$. The nitrogen hyperfine couplings, when included, had the following principal values $[98,16,17] \mathrm{MHz}$ expressed in the principal axis system (PAS) of the closeby electron g-tensor.

As introduced in Mentink-Vigier et al. [50, 54], we define the polarization gain $\epsilon_{B}$ as the ratio between the nuclear polarization with $\mu \mathrm{w}(\mu \mathrm{w})$ irradiation and the nuclear Boltzmann Polarization

$$
\epsilon_{B}=\frac{P_{n}(\mu \mathrm{w} \text { on })}{P_{n}(\text { Boltzmann })}
$$

the nuclear depolarization factor $\epsilon_{\text {Depo }}$ as the ratio between the nuclear polarization without $\mu \mathrm{w}$ irradiation and the nuclear Boltzmann Polarization

$$
\epsilon_{\text {Depo }}=\frac{P_{n}(\mu \mathrm{w} \text { off })}{P_{n}(\text { Boltzmann })}
$$

and the classical "DNP enhancement factor" $\epsilon_{\text {On/Off }}$ as the ratio of nuclear polarization with and without $\mu \mathrm{w}$ irradiation

$$
\epsilon_{\mathrm{On} / \mathrm{Off}}=\frac{P_{n}(\mu \mathrm{w} \text { on })}{P_{n}(\mu \mathrm{w} \text { off })}
$$

$$
\begin{aligned}
\widehat{H}(t) & =\widehat{H}_{0}(t)+\widehat{H}_{\mu \mathrm{w}} \\
\widehat{H}_{0}(t) & =\widehat{H}_{\mathrm{Z}}(t)+\widehat{H}_{\mathrm{HF}}(t)+\widehat{H}_{\mathrm{J}}+\widehat{H}_{\mathrm{Dip}}(t)+\widehat{H}_{\mathrm{d}}(t),
\end{aligned}
$$

with

$$
\begin{aligned}
\widehat{H}_{\mathrm{Z}}(t) & =\sum_{i}\left(g_{i}(t) \beta_{e} B_{0}-\omega_{\mu \mathrm{w}}\right) \widehat{S}_{i, z}-\sum_{n} \omega_{n} \widehat{I}_{n, z} \\
\widehat{H}_{\mathrm{HF}}(t) & =\sum_{i, n} A_{i, n}^{z}(t) \widehat{S}_{i, z} \widehat{I}_{n, z}+\frac{1}{2}\left(A_{i, n}^{+}(t) \widehat{S}_{i, z} \widehat{I}_{n}^{+}+A_{i, n}^{-}(t) \widehat{S}_{i, z} \widehat{I}_{n}^{-}\right) \\
\widehat{H}_{\mathrm{J}} & =\sum_{i<i^{\prime}}-2 J_{i, i^{\prime}}\left(\widehat{S}_{i, z} \widehat{S}_{i^{\prime}, z}+\frac{1}{2}\left(\widehat{S}_{i}^{+} \widehat{S}_{i^{\prime}}^{-}+\widehat{S}_{i}^{-} \widehat{S}_{i^{\prime}}^{+}\right)\right) \\
\widehat{H}_{\mathrm{Dip}}(t) & =\sum_{i<i^{\prime}} D_{i, i^{\prime}}(t)\left(2 \widehat{S}_{i, z} \widehat{S}_{i^{\prime}, z}-\frac{1}{2}\left(\widehat{S}_{i}^{+} \widehat{S}_{i^{\prime}}^{-}+\widehat{S}_{i}^{-} \widehat{S}_{i^{\prime}}^{+}\right)\right) \\
\widehat{H}_{\mathrm{d}}(t) & =\sum_{n<n^{\prime}} d_{n, n^{\prime}}(t)\left(2 \widehat{I}_{n, z} \widehat{I}_{n^{\prime}, z}-\frac{1}{2}\left(\widehat{I}_{n}^{+} \widehat{I}_{n^{\prime}}^{-}+\widehat{I}_{n}^{-} \widehat{I}_{n^{\prime}}^{+}\right)\right) \\
\widehat{H}_{\mu \mathrm{w}} & =\omega_{1} \sum_{i} \widehat{S}_{i, x} .
\end{aligned}
$$

where $\widehat{H}_{\mathrm{Z}}(t)$ stands for the Zeeman interaction of the spins, $\widehat{H}_{\mathrm{HF}}(t)$ for the secular and pseudo-secular part of the hyperfine coupling between the electrons and the nuclei, $\widehat{H}_{\mathrm{J}}$ for the exchange interaction between two electrons, $\widehat{H}_{\text {Dip }}(t)$ for both the secular and flipflop parts of the electron-electron dipolar interactions, $\widehat{H}_{\mathrm{d}}(t)$ for the homonuclear nuclear dipolar interaction, and $\widehat{H}_{\mu \mathrm{w}}$ for the $\mu \mathrm{w}$ irradiation. Using the notation $\omega_{i}=g_{i} \beta_{e} B_{0}$, we can describe the 4 rotor events induced by the sample spinning [51, 52, 54]:

- the $\mu \mathrm{w}$ rotor events, active when $\omega_{i}-\omega_{\mu \mathrm{w}}=0$, which induce changes in the electron polarization through the effect of $\widehat{H}_{\mu \mathrm{w}}$,

- the electron or nuclear dipolar rotor events, active when $\omega_{i / n}-\omega_{i^{\prime} / n^{\prime}}=0$, which induce 
exchanges of electron or nuclear polarizations through the action of the flip-flop of the dipolar coupling and exchange interaction when present,

- the CE rotor events, active when $\omega_{i}-\omega_{i^{\prime}} \approx \pm \omega_{n}$, which induce a exchange between the electron polarization difference and the proton polarization, through the combined effect of the flip-flop dipolar and pseudo-secular hyperfine couplings.

- the SE rotor events, active when $\omega_{i}-\omega_{\mu \mathrm{w}} \approx \pm \omega_{n}$, which induce a transfer of polarization between the electron and the proton, through the combined effect of the pseudo-secular hyperfine interaction and $\mu \mathrm{w}$ irradiation.

For a simplified three spin system $e_{a}-e_{b}-n$, equation 2 can be rewritten as:

$$
\begin{aligned}
\widehat{H}_{\mathrm{Z}}(t)= & \left(\omega_{a}(t)-\omega_{\mu \mathrm{w}}\right) \widehat{S}_{a, z}+\left(\omega_{b}(t)-\omega_{\mu \mathrm{w}}\right) \widehat{S}_{b, z} \\
& -\omega_{n} \widehat{I}_{n, z} \\
\widehat{H}_{\mathrm{HF}}(t)= & A_{z}(t) \widehat{S}_{a, z} \widehat{I}_{n, z} \\
& +\frac{1}{2}\left(A^{+}(t) \widehat{S}_{a, z} \widehat{I}_{n}^{+}+A^{-}(t) \widehat{S}_{a, z} \widehat{I}_{n}^{-}\right) \\
\widehat{H}_{\mu \mathrm{w}}= & \omega_{1}\left(\widehat{S}_{x, a}+\widehat{S}_{x, b}\right) \\
\widehat{H}_{\mathrm{J}}= & -2 J_{a, b}\left(\widehat{S}_{a, z} \widehat{S}_{b, z}+\frac{1}{2}\left(\widehat{S}_{a}^{+} \widehat{S}_{b}^{-}+\widehat{S}_{a}^{-} \widehat{S}_{b}^{+}\right)\right) \\
\widehat{H}_{\mathrm{Dip}}(t)= & D_{a, b}(t)\left(2 \widehat{S}_{a, z} \widehat{S}_{b, z}-\frac{1}{2}\left(\widehat{S}_{a}^{+} \widehat{S}_{b}^{-}+\widehat{S}_{a}^{-} \widehat{S}_{b}^{+}\right)\right)(3)
\end{aligned}
$$

A schematic illustration of the rotor events is provided in figure 1. We can observe the evolution of the electron and proton polarizations for the three main rotor events $(\mu \mathrm{w}$, dipolar and $\mathrm{CE})$. The effect of the $\mu \mathrm{w}$ and electron dipolar rotor events can be seen on the electron polarization curves whereas the effect of the $\mathrm{CE}$ rotor event appears too small to be seen on that scale. On the other hand the presence of CE rotor events is observed on the proton polarization curve. Note that the SE rotor events can not be observed in this simulation.

As described in previous work, the calculation of the spin evolution involves solving the time dependent (due to the sample spinning) master equation, including all necessary interactions and relaxation processes. This requires the computation of the propagator superoperator of a rotor period, using small stepwise integration and calculating this propagator superoperator $\widehat{\widehat{U}}_{\kappa}$ for every $\left(\kappa^{\text {th }}\right)$ rotor-step[52, 54]. For the three-spin system of two electrons and one nucleus the spin density matrix, representing the state of the system at any time $\widehat{\rho}(t)$ has a dimension of $2^{3} \times 2^{3}=64$ and is thus defined by 64 independent parameters. Thus in earlier work we presented the Liouville-von Neumann rate

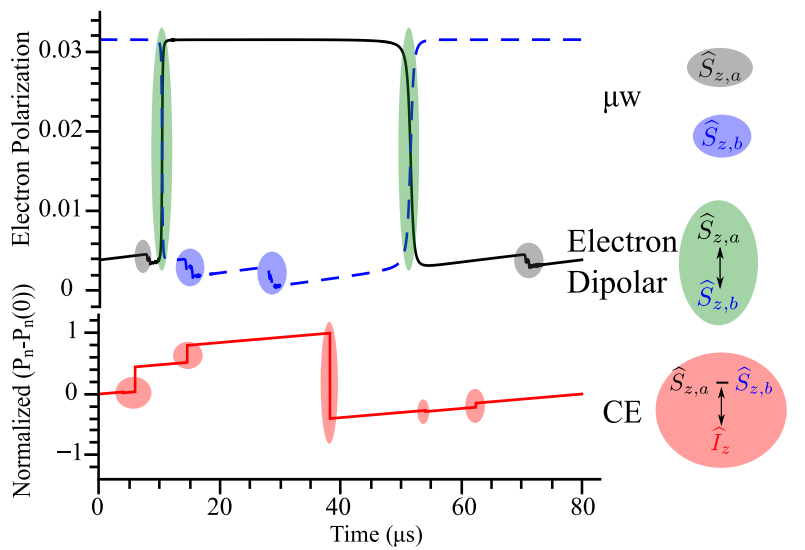

FIG. 1. Evolution of the electron and nuclear polarizations during a rotor period at quasi periodic steady-state for a single crystallite orientation. The black, blue (dashed) and red lines correspond to electron a, electron $\mathrm{b}$ and the nucleus respectively. Note that the nuclear polarization difference with respect to Boltzmann is normalized to observe the $\mathrm{CE}$ rotor events. The rotor events are labeled with different colors, grey corresponds to a $\mu \mathrm{w}$ rotor event on electron $a$, blue to a $\mu \mathrm{w}$ rotor event on electron $b$, green to an electron dipolar rotor event and red to a $\mathrm{CE}$ rotor event. On the right hand-side, the corresponding operators involved in the rotor events are displayed.

equation as a Liouville superoperator acting in the vector composed of all elements of $\widehat{\rho}(t)$. For the present study we replace this superoperator by a Liouville operator acting on the vector $\sigma(t)$ built of the coefficients $s^{(m)}(t)(m \in \llbracket 1,64 \rrbracket)$ of a set of 64 independent operators $\widehat{S}^{(m)}$ composing the spin density operator:

$$
\widehat{\rho}(t)=s^{(1)}(t) \widehat{E}+\sum_{m=2}^{64} 2 s^{(m)}(t) \widehat{S}^{(m)}
$$

where $\widehat{E}$ is the identity operator. In this expansion we choose operators with $\operatorname{Tr}\left(\left(\widehat{S}^{(m)}\right)^{2}\right)=1 / 2$ and therefore the elements of $\sigma(t)$ are the expectation values $\sigma^{(m)}=\operatorname{Tr}\left(\widehat{\rho} \widehat{S}^{(m)}\right)=1 / 2$. The large dimension, and the time dependence of the evolution operator are the main cause of the long time necessary to solve the master equation. Typically, using a full Liouville calculation, and a 3 spin system, a powder-averaged 30 points field-sweep was simulated in 6 to 8 hours. Therefore larger spin systems are not currently accessible: a 5 spin simulation takes about 1 day per crystallite orientation. The duration of such simulations prohibits in-depth analysis (that would require scanning through multiple parameters) or the extension to much larger and therefore realistic spin systems.

In this work, we achieve a drastic reduction of the vector size $\sigma$ to shorten significantly the computational time. To that purpose, we assume that the spin dy- 


$$
\widehat{\rho}(t)=s_{0}(t) \widehat{E}+\sum_{p=x, y, z} 2 s_{p}^{12}(t) \widehat{S}_{p}^{(12}
$$

m

namics are well described by a series of successive rotor events, with each corresponding to a two-level anticrossing problem,along with spin-lattice relaxation towards Boltzmann equilibrium. As discussed in the following section, each rotor event is treated/computed using either Bloch-type derivation in a reduced Liouvillian subspace for which relaxation can be simply introduced or with the LZ formalism (accounting for the variation in polarization operator). In between rotorsevents, the spin dynamics are well described by the secular part of the full Hamiltonian (see Eq. 3) and correspond to a return to Boltzmann equilibrium. In the following, section A describes the general approach to treat and approximate the spin dynamics in between rotor events and during each individual rotor event. Section B and C describe the computation of a propagator superoperator over a rotor period when all rotor events are treated with the LZ formalism or using a combination of the LZ and Bloch-type formalisms, respectively. Finally section D describes the extension to large spin assemblies (multiple electrons and protons).

\section{A. Independent diabatic rotor events - The Bloch-type approach and rotor synchronized propagation}

Every rotor event previously described involves only two energy levels. Therefore in the following we assume that rotor events, which correspond to diabatic passages, are well separated and independent. For a given rotor event involving the two levels $|1\rangle$ and $|2\rangle$, one can derive an effective Hamiltonian of the form:

$$
\begin{aligned}
\widehat{H}_{12}(t) & =\Delta \omega_{12}(t) \widehat{S}_{z}^{12}+\xi_{x}^{12}(t) \widehat{S}_{x}^{12}+\xi_{y}^{12}(t) \widehat{S}_{y}^{12}, \\
& =\frac{1}{2}\left(\begin{array}{cc}
\Delta \omega_{12}(t) & \xi_{12}(t) \\
\xi_{12}^{*}(t) & -\Delta \omega_{12}(t)
\end{array}\right)
\end{aligned}
$$

Where $\xi_{12}(t)=(1 / 2)\left(\xi_{x}^{12}-i \xi_{y}^{12}\right)$ and $\widehat{S}_{x}^{12}, \widehat{S}_{y}^{12}, \widehat{S}_{z}^{12}$ are the fictitious spin- $1 / 2$ operators corresponding to the transition $|1\rangle-|2\rangle . \Delta \omega_{12}$ and $\xi_{12}$ are the time dependent energy difference between the two states $|1\rangle$ and $|2\rangle$ and the magnitude of matrix element connecting them, respectively. In the absence of relaxation we can derive a Liouville superoperator operating on the $\sigma$ vector composed of the coefficients $\left\{s_{z}^{12}(t), s_{y}^{12}(t), s_{x}^{12}(t)\right\}$ of the spin density operator expansion

resulting in the Liouville-von Neumann equation:

$$
\frac{\mathrm{d} \sigma}{\mathrm{d} t}=\widehat{\widehat{L}}_{H}^{1,2}(t) \sigma
$$

with

$$
\widehat{\widehat{L}}_{H}^{1,2}(t)=\left[\begin{array}{ccc}
0 & -\xi_{x}^{12}(t) & \xi_{y}^{12}(t) \\
\xi_{x}^{12}(t) & 0 & -\Delta \omega^{12}(t) \\
-\xi_{y}^{12}(t) & \Delta \omega^{12}(t) & 0
\end{array}\right]
$$

373 which corresponds to homogeneous linear first-order differential equations (also known as the Bloch equations) for which the density matrix can easily be computed. The relaxation can also be efficiently introduced while keeping a homogeneous set of equations $[62,63]$ by increasing the matrix dimension by one unit. In this case, the vector vector $\sigma$ is extended to $\left\{1, s_{z}^{12}(t), s_{y}^{12}(t), s_{x}^{12}(t)\right\}$ and the $4 \times 4$ Liouvillian takes the form of a homogeneous Bloch operator:

$$
\widehat{\widehat{L}}_{B}^{1,2}(t)=\left[\begin{array}{cccc}
0 & 0 & 0 & 0 \\
s_{z}^{1,2, e q} / T_{1} & -1 / T_{1} & -\xi_{x}^{12}(t) & \xi_{y}^{12}(t) \\
0 & \xi_{x}^{12}(t) & -1 / T_{2} & -\Delta \omega^{12}(t) \\
0 & -\xi_{y}^{12}(t) & \Delta \omega^{12}(t) & -1 / T_{2}
\end{array}\right]
$$

382 from which the propagator of a short time interval $[\kappa \delta t,(\kappa+1) \delta t]$ can be obtained via $\widehat{\widehat{U}}_{\mathrm{B}, \kappa}^{1,2}=$ $\exp \left(\widehat{\widehat{L}}_{B}^{12}(\kappa t) \delta t\right)$.

Following this approach, the complete derivation for each type of rotor event can be obtained (see Supporting Information). The full Liouvillian superoperator accounting for all types of rotor events - can then be obtained by applying the superposition principle since the rotor events are assumed to be independent. In the end, the spin dynamics can be described by the evolution of a $\sigma$ vector with dimension 18 and thus by a 18 $\times 18$ Liouvillian superoperator. The elements of $\sigma(t)$ in this case become the 18 prefactors of the operators $\left\{\widehat{E}, \widehat{S}_{a, z}, \widehat{S}_{a, y}, \widehat{S}_{a, x}, \widehat{S}_{b, z}, \widehat{S}_{b, y}, \widehat{S}_{b, x}, \widehat{S}_{\mathrm{ZQ}, y}^{\mathrm{DJ}}, \widehat{S}_{\mathrm{ZQ}}^{\mathrm{DJ}} \widehat{I}_{n, z}\right.$, $\left.\widehat{S}_{y}^{\mathrm{CE}+}, \widehat{S}_{x}^{\mathrm{CE}+}, \widehat{S}_{y}^{\mathrm{CE}-}, \widehat{S}_{x}^{\mathrm{CE}-}, \widehat{S}_{y}^{\mathrm{DQ} a}, \widehat{S}_{x}^{\mathrm{DQ} a}, \widehat{S}_{y}^{\mathrm{ZQ} a}, \widehat{S}_{x}^{\mathrm{ZQ} a}\right\}$, (see Supporting Information for the derivation details). 398 This constitute a drastic size reduction compared to $64 \times 64$ in the full Liouville approach that results in massive time savings with minimal compromise on the accuracy (as demonstrated in the rest of the paper). As described previously,[52,54] the rotor synchronized propagator is simply obtained by step integration over one rotor period:

$$
\widehat{\widehat{U}}_{\text {rotor }}=\prod_{\kappa=1}^{N_{s}} \widehat{\widehat{U}}_{\kappa}
$$

406

406 407 408 409 410 where $N_{s}$ stands for the number of integration points, and $\widehat{\widehat{U}}_{\kappa}=\exp \left(\widehat{\widehat{L}}_{\kappa} \times \delta t\right)$ with $\delta t=1 /\left(N_{s} \nu_{r}\right)$ and $\widehat{\widehat{L}}_{\kappa}=$ $\widehat{\widehat{L}}_{B}(\kappa \delta t)$.

Note that in previous MAS-DNP calculations, the relaxation times $T_{1}$ and $T_{2}$ were determined after diagonalization of the Hamiltonian (without the $\mu \mathrm{w}$ term). 
As a result the $T_{1}$ and $T_{2}$ relaxation values were not constant during sample rotation because of the strong state mixing that occurs during the D-J and CE rotor events. On the contrary, with the Bloch-type approach presented in this work, we assume such relaxation times to be constant in order to optimize the computational performance. Such an approximation can be justified by the fact that the duration of state mixing is shorter than the electron $T_{2}$ in most cases and that the coherences created during the events decay after the mixing period. As shown below, this is further validated by the very good agreement with full Liouville calculations. More details can be found in the SI.

Accounting for relaxation effects is especially important when probing cases with large $\omega_{1}$ and/or short electronic $T_{2}$. This is notably also the case for strong $\mu \mathrm{w}$ irradiation strength, large dipolar and/or J interactions, radicals with narrow EPR lines such as Trityl, and short electron relaxation times. In all other cases, the Bloch type treatment can be simplified using the LZ approximation.

\section{B. Independent diabatic rotor events - Combining Landau-Zener approximation and rotor synchronized propagation}

Coming back to the two level system as defined by the Hamiltonian in eq. 5 we can follow Vitanov [64] and define a scaled dimensionless coupling parame-
439 ter derived from $\xi_{12}(t), \omega_{12}\left(t_{\times}\right)=\xi_{12}\left(t_{\times}\right) / \beta_{12}$ and ${ }_{440} \quad \beta_{12}=\sqrt{\left(\frac{d \Delta \omega_{12}(t)}{d t}\right)_{t_{x}}}$ and a time parameter $\tau_{12}=\beta_{12} t$. 441 For rotor events where $\omega_{12}^{2} \ll 1$, the LZ approxima442 tion, which gives the variation of population across a 443 resonant condition (diabatic passage), can be safely ap444 plied and the transition jump time $\tau_{\text {jump }}[64]$ is about 445 constant and equal to $\sqrt{2 \pi}$, [64] which translates to a 446 jump time of $\sim 1 \mu \mathrm{s}$ or smaller, as compared to the 447 rotor period which is typically about $10-1000 \mu \mathrm{s}$. In 448 this case, assuming that levels $|1\rangle$ and $|2\rangle$ cross at time $449 t_{\times}$, the LZ formula, expressing the changes in popula450 tions, can be formulated as the change in the coefficient ${ }^{451}$ of the the $\widehat{S}_{z}^{1,2}$ ) operator in the spin density operator 452 expansion in eq. 6 . This translates onto $s_{z}^{1,2}$ as:

$$
s_{z}^{1,2}\left(t_{\times}^{+}\right)=\left[1-2 \epsilon^{12}\right] s_{z}^{1,2}\left(t_{\times}\right)
$$

453 with

$$
\epsilon^{12}=1-\exp \left[\frac{-\pi\left|\xi_{12}\left(t_{\times}\right)\right|^{2}}{2\left|\frac{\mathrm{d}}{\mathrm{d} t} \Delta \omega_{12}\right|_{t_{\times}}}\right] .
$$

${ }_{454}$ Here $t_{\times}^{+}$is the time just after the crossings. As the 455 calculation are performed by step integration, we as${ }_{456}$ sume that $t_{\times}$and $t_{\times}^{+}$are within a time step interval ${ }_{457}\left[t_{\kappa}, t_{\kappa}+\delta t\right]$. When a crossing occurs we then assume 458 that $t_{\kappa} \cong t_{\times}$and that the propagator for the time 459 interval $\left[t_{\kappa}, t_{\kappa+1}\right]$ can be written in the reduced basis $460\left\{1, s_{z}^{12}\right\}$ as follows:

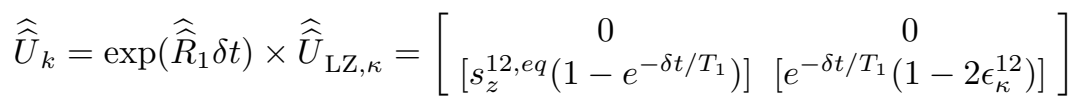

If there is no crossing during this interval, then $\widehat{\widehat{U}}_{\mathrm{LZ}, \kappa}$ is identity.

On the three-spin system the rotor events usually occur at different time-steps, and in the following we assume that they can be successively treated. This allows to rely on a LZ formulae for each rotor event separately. For each type of event one can identify the two levels involved in the diabatic passage and define the coefficient $s_{z}^{(m)}$ of the $z$-operator $\widehat{S}_{z}^{(m)}$ that changes at this passage. As is shown in the SI, the changes in all of these coefficients can be transferred to changes in only the coefficients $\left\{s_{a, z}, s_{b, z}, s_{n, z}\right\}$ of the operators $\left\{\widehat{S}_{z, a}, \widehat{S}_{z, b}, \widehat{I}_{z, n}\right\}$. Adding relaxation, the Liouville superoperator representing all events while relying on LZ formula, operates on the vector $\left\{1, s_{a, z}, s_{b, z}, s_{n, z}\right\}$ and the propagator at step $\kappa$ can be written in the basis $\widehat{E}$,
${ }_{477} \widehat{S}_{z, a}, \widehat{S}_{z, b}, \widehat{I}_{z, n}$ as follows:

$$
\widehat{\widehat{U}}_{\kappa}=\exp \left(\widehat{\widehat{R}}_{1}(\kappa \delta t) \times \delta t\right) \widehat{\widehat{U}}_{\mathrm{LZ}, \kappa}^{\mu \mathrm{w}} \widehat{\widehat{U}}_{\mathrm{LZ}, \kappa}^{\mathrm{D}-\mathrm{J}} \widehat{\widehat{U}}_{\mathrm{LZ}, \kappa}^{\mathrm{CE}} \widehat{\widehat{U}}_{\mathrm{LZ}, \kappa}^{\mathrm{SE}}
$$

478 where $\widehat{\widehat{R}}_{1}$ represents the longitudinal relaxation dur479 ing the time interval $\left[t_{\kappa}, t_{\kappa}+\delta t\right]$ and its $4 \times 4$ evolution 480 operator has the form:

$$
\widehat{\widehat{R}}_{1}=\left[\begin{array}{cccc}
1 & 0 & 0 & 0 \\
s_{a, z}^{e q} / T_{1, a}^{e} & -1 / T_{1, a}^{e} & 0 & 0 \\
s_{b, z}^{e q} / T_{1, b}^{e} & 0 & -1 / T_{1, b}^{e} & 0 \\
s_{n, z}^{e q} / T_{1}^{n} & 0 & 0 & -1 / T_{1}^{n}
\end{array}\right]
$$

481 The explicit forms of the Liouvillians in Eq. 14 are 482 given in the SI. As above, the rotor synchronized prop483 agator is obtained by step integration over one rotor 
period:

$$
\widehat{\widehat{U}}_{\text {rotor }}=\prod_{\kappa=1}^{N_{s}} \widehat{\widehat{U}}_{\kappa}
$$

The novelty here relies on the fact that the LZ formula are applied to the $z$-coefficients of the spin density operator expansion and not on the populations of the spin system, which allows deriving a rotorsynchronized evolution operator while accounting for relaxation. As discussed in the previous section, the simulation code is much faster than the full Liouville calculation thanks to the reduced matrix dimensions. This LZ treatment is appropriate when the rotor events can be considered infinitely sharp. When the diabatic passages at the rotor events cannot be described accurately enough by the LZ formalism, because their corresponding $\omega_{\mathrm{RE}}(\mathrm{RE}=\mathrm{DJ}, \mathrm{CE}, \mathrm{SE})$ parameters are greater than 1, then their Liouville superoperators

$\widehat{\widehat{U}}_{\mathrm{LZ}, \kappa}^{\mathrm{RE}}$ must be replaced by the Bloch-type of operators $\widehat{\widehat{U}}_{B \kappa}^{R E}$.

C. Combining Bloch-type and Landau-Zener formalism with rotor synchronized propagation

For diabatic passage where $\omega_{12}^{2} \gg 1$, it is important to stress that the LZ formula only gives a crude approximation of the variation in population. This is potentially the case for $\mu \mathrm{w}$ and electron dipolar rotor events when dealing with strong $\mu \mathrm{w}$ irradiation, large electron dipolar interactions, or radicals with narrow
EPR lines. In such cases, the dimensionless jump time can be approximated by $\tau_{\text {jump }}=2 \omega_{12}$, which translates to a jump time greater than $1 \mu \mathrm{s}$, [64] i.e. much larger than the integration step $\delta t$ and potentially even larger than the corresponding electron/nuclei relaxation times. In such cases the Bloch-type formalism, as described above, should be preferred since it allows accounting for longitudinal but also transverse relaxation effects during rotor events. Furthermore, for very short $T_{2}^{e}(\sim \mu \mathrm{s})$ values, possibly leading to off-resonance and saturation effects when $\omega_{1} / 2 \pi$ and $1 / T_{2}^{e}$ are in the $\mathrm{MHz}$ range or larger, we must also turn to the Bloch approach.

On the other hand, the LZ treatment can be safely applied for SE and CE rotor events (as well as intermolecular electron dipolar rotor events, see section $\mathrm{D}$ ) for which $\omega_{\mathrm{CE} / \mathrm{SE}}$ is always much smaller than 1 and the associated jump time is much smaller than the $\mu \mathrm{s}$ timescale. In addition $T_{2}^{n}$ and $T_{2}^{e}$ are usually long enough to be safely neglected during the SE and CE crossings.

Here we propose to combine both approaches: i.e. to treat $\mathrm{SE}$ and $\mathrm{CE}$ rotor events with the LZ approach and the $\mu \mathrm{w}$ and D-J rotor events using "Bloch-type" derivations. For a two electrons and one nucleus spin system, this can be done resulting in a Liouville superoperator of dimension $10 \times 10$ operating on the vector $\left\{1, s_{z, a}, s_{y, a}, s_{x, a}, s_{z, b}, s_{y, b}, s_{x, b}, s_{y, \mathrm{ZQ}}^{\mathrm{D}-\mathrm{J}}, s_{z, \mathrm{ZQ}}^{\mathrm{D}-\mathrm{J}}, s_{z, n}\right\}$ where the LZ part of the CE and SE events has matrix elements only between $\left\{s_{z, a}, s_{z, b}, s_{z, n}\right\}$ and the Bloch part of the $\mu \mathrm{w}$ and D-J events has the form

$$
\widehat{\widehat{L}}_{B}(t)=\left[\begin{array}{cccc}
0 & 0 & 0 & 0 \\
s_{a, z}^{e q}(t) / T_{1}^{e} & -1 / T_{1}^{e} & \omega_{1} & 0 \\
0 & -\omega_{1} & -1 / T_{2}^{e} & -\Delta \omega_{\mathrm{a}}(t) \\
0 & 0 & -\Delta \omega_{\mathrm{a}}(t) & -1 / T_{2}^{e} \\
s_{b, z}^{e q}(t) / T_{1}^{e} & 0 & 0 & 0 \\
0 & 0 & 0 & 0 \\
0 & 0 & 0 & 0 \\
0 & -D J_{a b}(t) / 2 & 0 & 0 \\
0 & 0 & 0 & 0 \\
s_{n, z}^{e q} / T_{1}^{n} & 0 & 0 & 0
\end{array}\right.
$$

where we defined a transverse relaxation time for the D-J rotor events $T_{2, \mathrm{ZQ}}^{e}=T_{2}^{e} / 2$ and $D J_{a b}=\left(D_{a, b}+\right.$ $\left.2 J_{a, b}\right)$ (See SI for details). The time-step integration is still used to obtain the periodic propagator

$$
\widehat{\widehat{U}}_{\text {rotor }}=\prod_{\kappa=1}^{N_{s}} \widehat{\widehat{U}}_{\kappa}
$$

544

and at each time-step $\kappa$, the propagator is the product

$\left.\begin{array}{cccccc}0 & 0 & 0 & 0 & 0 & 0 \\ 0 & 0 & 0 & D J_{a b}(t) & 0 & 0 \\ 0 & 0 & 0 & 0 & 0 & 0 \\ 0 & 0 & 0 & 0 & 0 & 0 \\ -1 / T_{1}^{e} & \omega_{1} & 0 & -D J_{a b}(t) & 0 & 0 \\ -\omega_{1} & -1 / T_{2}^{e} & -\Delta \omega_{\mathrm{b}}(t) & 0 & 0 & 0 \\ 0 & -\Delta \omega_{\mathrm{b}}(t) & -1 / T_{2}^{e} & 0 & 0 & 0 \\ D J_{a b}(t) / 2 & 0 & 0 & -1 / T_{2, \mathrm{ZQ}}^{e} & -\Delta \omega_{\mathrm{D}}(t) & 0 \\ 0 & 0 & 0 & \Delta \omega_{\mathrm{D}}(t) & 0 & 0 \\ 0 & 0 & 0 & 0 & 0 & -1 / T_{1}^{n}\end{array}\right]$

${ }_{545}$ between the Bloch and LZ part

$$
\widehat{\widehat{U}}_{\kappa}=\widehat{\widehat{U}}_{\mathrm{B}, \kappa} \widehat{\widehat{U}}_{\mathrm{LZ}, \kappa}
$$

546 where $\widehat{\widehat{U}}_{\mathrm{B}, \kappa}=\exp \left(\widehat{\widehat{L}}_{B}(\kappa \delta t) \times \delta t\right)$, and $\widehat{\widehat{U}}_{\mathrm{ZL}, \kappa}=\widehat{\widehat{U}}_{\mathrm{SE}, \kappa} \times$ $\widehat{\widehat{U}}_{\mathrm{CE}, \kappa}$. This (Hybrid) approach ensures accurate sim${ }_{548}$ ulations even for short electron $T_{2}^{e}$ (i.e $<2 \mu$ s for ni${ }^{549}$ troxides at $9 \mathrm{~T}$ ), for large $\omega_{\mathrm{RE}}$ interactions (e.g strong ${ }_{550} \mu \mathrm{w}$ fields) or narrow EPR line widths (e.g in the case 551 of Trityl). 
D. MAS-DNP simulation of large spin ensembles 600 (tens to thousands of spins): extension to multiple 601 electrons and nuclei

Here we extend our three-spin system by adding many electrons and nuclei with the aim to generate more realistic simulations able to reproduce the electron concentration and nuclear spin diffusion effects found in contemporary experiments. The presence of intermolecular electron-electron dipolar interactions generates a MAS-induced spectral diffusion phenomenon which tends to equilibrate the polarization throughout the EPR line,[53] and directly impacts the intramolecular polarization difference and thus the overall nuclear polarization enhancement. Moreover, the presence of additional nuclei induces new CE rotor events and tends to equilibrate the polarization among the nuclei. In order to meet both challenges, two models and codes were developed and are described below.

\section{Increasing the number of electron spins: accounting} for the electron concentration effect (the box model)

We simulate $N$ biradicals (each modeled by 2 electrons and 1 proton) randomly distributed in a box so as to meet a given biradical concentration. In this model, referred as the box model in this work, interactions between biradicals are restricted to nearest neighbors. This approximation allows keeping an efficient computational code while accounting for intermolecular dipolar rotor events, i.e. electron spectral spin diffusion, in a similar fashion as nuclear spin diffusion process $[65,66]$. A similar approach was previously described by Thurber et al. [53]. During standard DNP experiments, the biradical concentration is $\sim 5-30$ $\mathrm{mM}$ which translates to $\sim 0.3-1.5 \mathrm{MHz}$ and $0.45-2$ $\mathrm{kHz}$ of intermolecular dipolar and hyperfine couplings respectively. These additional interactions induce intermolecular rotor events (dipolar, CE, SE) that can efficiently be computed using the LZ computational approach. More precisely, the code generates $\mathrm{N}$ copies of a 3 spin system (2 electrons and one nucleus) with a fixed configuration, which are randomly dispersed and oriented in a box.

In order to allow the use of the LZ approach, some constraints must be applied:

- Two identical crystallite orientations cannot coexist within the same box (in order to avoid frequency degeneracy).

- The intermolecular electron-electron distance $\left(d_{\text {min }}\right)$ is always larger than $1.7 \mathrm{~nm}(\sim 10 \mathrm{MHz}$ dipolar coupling) so that LZ can be applied accurately.

- For simplicity, the effects on the spin system of the dipolar rotor events between electrons that are more than $d_{\max }=6.4 \mathrm{~nm}(\sim 0.2 \mathrm{MHz}$ dipolar coupling) are ignored.

The calculation is then modified in order to account for intermolecular rotor events. For a given integration step, the propagator is now written:

$$
\widehat{\widehat{U}}_{\kappa}=\widehat{\widehat{U}}_{\mathrm{B}, \kappa} \times \widehat{\widehat{U}}_{\mathrm{LZ}, \kappa}
$$

where where $\widehat{\widehat{U}}_{\mathrm{B}, \kappa}$ is obtained from the previous section, and $\widehat{\widehat{U}}_{\mathrm{LZ}, \kappa}=\widehat{\widehat{U}}_{\mathrm{LZ}, \kappa}^{\mathrm{SE}, \text { inter }} \times \widehat{\widehat{U}}_{\mathrm{LZ}, \kappa}^{\mathrm{CE} \text {,inter }} \times \widehat{\widehat{U}}_{\mathrm{LZ}, \kappa}^{\mathrm{SE} \text {,intra }} \times$ $\widehat{\widehat{U}}_{\mathrm{LZ}, \kappa}^{\mathrm{CE} \text {,intra }}$. Thus the overall $\widehat{\widehat{U}}_{\kappa}$ has a dimension of $10 N \times 10 N$, scaling linearly with $N$.

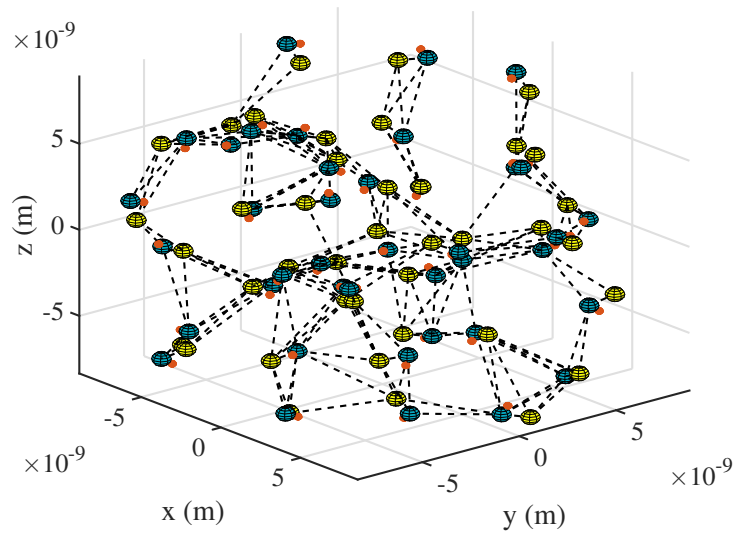

FIG. 2. Example of a spin system used in the box model corresponding here to a random distribution of $N=40$ biradicals (15 mM concentration). In blue electron of type "a", yellow, electron of type "b", orange, nucleus. The black dotted lines correspond to the intermolecular electron-electron dipolar couplings active during the simulation.

Figure 2 shows a typical random distribution generated by the code: 40 biradicals with $15 \mathrm{mM}$ concentration, with $d_{\min }=1.7 \mathrm{~nm}$ and $d_{\max }=6.4 \mathrm{~nm}$. Note that the box model presented here excludes the possibility of having two coupled biradicals with exactly the same orientation since it would complicate exact simulations and that this possibility is highly improbable.

Numerical stability of the output can be improved by increasing the number of biradicals and/or averaging the results over several randomly generated boxes. ${ }_{623} 40$ to 50 biradicals, averaged over 10 different boxes, ${ }_{624}$ is sufficient to achieve $<5 \%$ stability. Typically, the 625 computation of the propagator (eq. 18) for 40 biradi626 cals takes about 100 to 170 seconds (on a single CPU 
thread) for the full LZ or the Hybrid approach respec- 675 tively. In the end, a 30 point field sweep profile can be 676 computed in 20 minutes for quick tests (averaging over 677 4 boxes) or in about 1 hour for an accurate computa- ${ }^{678}$ tion (averaging over 10 boxes).

\section{Including additional nuclei}

The introduction of additional (hyperfine coupled) nuclei leads to more $\mathrm{CE} / \mathrm{SE}$ rotor events but also to a new type of event, the nuclear-nuclear dipolar rotor event, $[51,54]$ which occurs when two nuclei with different hyperfine couplings have the same instantaneous resonant frequency. These rotor events allow hyperpolarized nuclei close to the unpaired electrons to propagate their polarizations to more distant spins. This MAS-dependent effect has been accounted for theoretically [see ref [54] for details] to predict a reduction/removal of the so-called diffusion barrier present in the static case [67-71]. As in the electron spins' case, the nuclear dipolar rotor events induce a partial exchange of the nuclear polarization which can be accurately computed using the LZ derivation. Such a treatment is applied to "local nuclei" (also called closeby or ENDOR nuclei) for which hyperfine couplings are larger than the mean nuclei-nuclei dipolar interaction. In addition we can refine our model and add additional "bulk" nuclei, which are not directly coupled to the electrons but are in contact with some of the "local nuclei". The nuclear spin diffusion among the bulk nuclei is simulated using rate equations that equilibrates the polarization between two connected nuclei $[66,72,73]$ and for nucleus $j$ is given by

$$
\frac{\mathrm{d} s_{z, j}}{\mathrm{~d} t}=\sum_{j^{\prime}}-r_{j, j^{\prime}}^{\mathrm{SD}}\left(s_{z, j}-s_{z, j^{\prime}}\right)+\frac{s_{z, j}^{e q}}{T_{1, j}}
$$

where $j^{\prime}$ corresponds to the index of the neighboring nucleus, and $r_{j, j^{\prime}}^{\mathrm{SD}}=d_{j, j^{\prime}}^{2} T_{2}^{n} / 4$. Hence the assumption used to build our model containing two electrons $\left(e_{a}, e_{b}\right)$ and $N_{n}$ nuclei can be listed as follows:

- CE rotor events involving "local" nuclei occur when $\left|\omega_{a}-\omega_{b}\right| \approx \omega_{n}$. Each CE rotor event, involving a given nucleus, can be a priori treated using the derivation provided in the SI for a 3 spin case. In addition this CE rotor event condition is a priori also influenced by the presence of other hyperfine coupled nuclei which induce splittings of the effective electron resonance, leading to a quasi-continuum of $\mathrm{CE}$ sub-conditions. Overall these additional splittings can be safely ignored since they all contribute identically to the CE polarization transfer and it simplifies the treatment.
686 687

- The local nuclei are connected among themselves via nuclear dipolar rotor events. The LZ approach can be used safely here to describe the energy crossing with $\Delta \omega_{0}=\left(A_{z, 1}-A_{z, 2}\right) / 2$.

- Couplings among "bulk" nuclei are introduced through a semi-classical spin-diffusion treatment.

- The last shell of the Local nuclei are connected to bulk nuclei via nuclear dipolar rotor events.

- The evolution operator at each step can thus be written as

$$
\widehat{\widehat{U}}_{\kappa}=\widehat{\widehat{U}}_{\mathrm{B}, \kappa} \times \widehat{\widehat{U}}_{\mathrm{LZ}, \kappa}
$$

where

$$
\widehat{\widehat{U}}_{\mathrm{LZ}, \kappa}=\prod_{j=1}^{N_{L}} \widehat{\widehat{U}}_{\mathrm{LZ}, \kappa}^{\mathrm{CE}, \mathrm{j}} \times \prod_{j=1}^{N_{L}} \widehat{\widehat{U}}_{\mathrm{LZ}, \kappa}^{\mathrm{SE}, j} \times \prod_{j=1}^{N_{L}} \prod_{j^{\prime}=n}^{N_{L}} \widehat{\widehat{U}}_{\mathrm{ZL}, \kappa}^{\mathrm{dip}, j, j^{\prime}}
$$

and where the Bloch part accounts for electrons' dynamic (dipolar and $\mu \mathrm{w}$ rotor events), relaxation and the semi-classical spin diffusion among the Bulk nuclei. To perform the simulations, the code generates a partially random distribution of nuclei. The local nuclei $N_{L}$ are only connected to the electron $a$, and distributed within a cone shape of variable solid angle. Within this cone, the nuclei are arranged in layers and the nuclei are spaced by a mean distance that corresponds to a given nuclei concentration. The choice of the cone geometry was driven by computational tradeoff and simplicity. In particular the cone angle chosen allows computing a large number of local nuclei while keeping the computational time reasonable. The nuclei in the cone are only coupled to one electron here which allows relating their positioning (i.e. distance to electron) to the polarization transfer efficiency. Nevertheless, the code could easily be modified to account for couplings to both electrons, since only $\mathrm{CE}$ rotor events are impacted.[74-76] This would provide more accurate simulations but is beyond the scope of the current article.

Figure 3 shows a typical cone distribution. The positions of "bulk" nuclei are not computed, instead the dipolar couplings between nearest neighbors are considered equal to an average value. More precisely, each nucleus is connected to six partners with a corresponding semi-classical rate defined by the mean nuclearnuclear dipolar coupling and the $T_{2}^{n}$. This geometry is basically the same for all the simulations presented in this manuscript where we probed the influence of changes in parameters such as nuclear relaxation times, electron-electron dipolar interactions, and hyperfine coupling to the closest nuclei. The interplay between the geometry of the local and bulk nuclei and the DNP efficiency will be investigated in future work. 


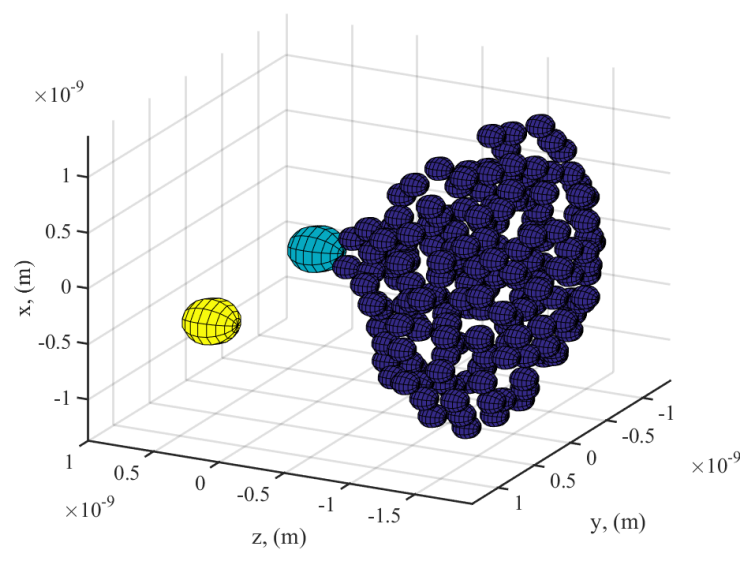

FIG. 3. Example of spin system used in the bulk model: here $N_{L}=182$ local nuclei were randomly generated. The blue sphere corresponds to electron of type "a", the yellow sphere to electron of type "b", and the dark blue spheres to local nuclei.

In the simulations presented below, the two closest local protons have a hyperfine coupling in frequency units that ranges between 1 and $4 \mathrm{MHz}$, whereas the farthest local nuclei have a hyperfine coupling of about 16 - $20 \mathrm{kHz}$. The mean nuclear dipolar coupling between all protons was equal to $1.7 \mathrm{kHz}$ (considering a $20 \mathrm{M}$ proton concentration). The two electrons used in the model have geometries and interactions similar to the biradical TOTAPOL $[50,52,54]$. The system's \{biradical + nuclei $\}$ orientation with respect to the magnetic field has been averaged using $144 \mathrm{ZCW}$ crystal orientations, and to account for the electron $T_{2}^{e}$ the Hybrid approach has been used. The nuclear $T_{2}^{n}$ is only added to account for the the spin diffusion. In all the simulations, $T_{2}^{n}$ was set to $10 \mathrm{~ms}$ and it's worth noting that an increase up to $200 \mathrm{~ms}$ did not significantly change the results.

\section{ON THE ACCURACY OF THE NEW CODE COMPARED TO FULL LIOUVILLE SIMULATIONS}

\section{A. Comparison between Liouville, LZ and the Hybrid approaches}

To assess the relevance of the approximations presented above, the full Liouville, Hybrid and LZ methods were compared in the 3 spin case. The spin system was built around a TOTAPOL-like geometry, and the calculations performed using standard spin relaxation properties along with powder averaging over 144 ZCW orientations. The temperature was fixed at 100 $\mathrm{K}$ (defining the thermal equilibrium), $B_{0}=9.394 \mathrm{~T}$, $\omega_{\mu \mathrm{w}} / 2 \pi=263.45 \mathrm{GHz}$, and $\omega_{1} / 2 \pi=0.85 \mathrm{MHz}$, and except where otherwise specified the MAS frequency was $\omega_{r} / 2 \pi=8 \mathrm{kHz}$.

$\epsilon_{B}$ at steady-state has been calculated as a function of the MAS frequency. Several electron dipolar coupling strength were tested for all three methods.
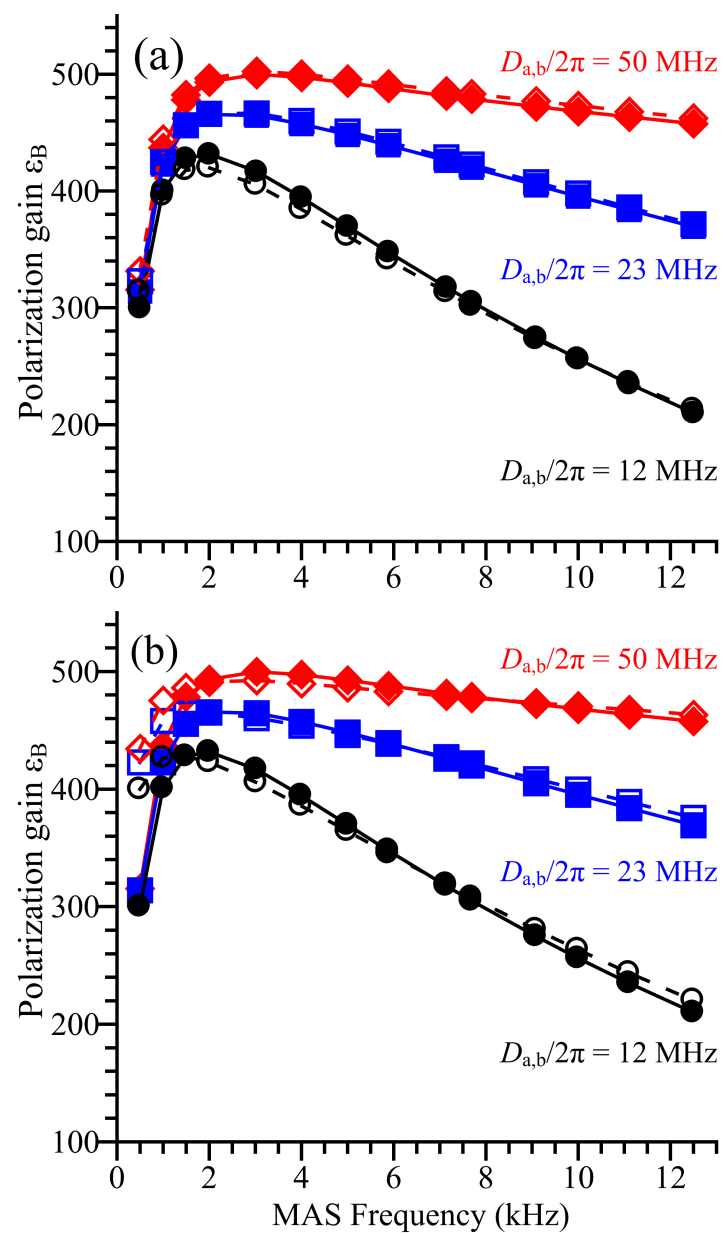

FIG. 4. (Three spins (e-e-n) simulations) (a) MAS dependence of $\epsilon_{B}$ for full Liouville calculations (full lines) and the Hybrid model (dashed lines) after powder averaging, (b) for full Liouville calculations (full lines) and LZ model (dashed lines) after powder averaging, computed for different dipolar interaction strength: $D_{a, b} / 2 \pi=12 \mathrm{MHz}$ (black circles), $D_{a, b} / 2 \pi=23 \mathrm{MHz}$ (blue squares), $D_{a, b} / 2 \pi=50 \mathrm{MHz}$ (red diamond). For all the simulations, $T_{1}^{e}=0.3 \mathrm{~ms}, T_{1}^{n}=4$ $\mathrm{s}, A_{1, a} / 2 \pi=1.5 \mathrm{MHz}$ and $T_{2}^{e}=1 \mu \mathrm{s}$ (full Liouville and Hybrid model), $T_{2}^{n}=0.2 \mathrm{~ms}$ (full Liouville model).

In figure 4 (a) and (b), solid lines are the complete Liouville calculations, dashed lines are respectively the Hybrid method in (a), and LZ method in (b). In the full Liouville model $\epsilon_{B}$ increases as the MAS frequency is increased up to $2-3 \mathrm{kHz}$, then after a maximum, $\epsilon_{B}$ decreases highlighting a reduction of the DNP mechanism efficiency. This loss is more drastic in the case 
of weak dipolar couplings compared to larger ones, a behavior that was previously explained [54], and could be summarized as: the MAS frequency is increased, the dipolar rotor events do not succeed in maintaining a large polarization difference between the electrons $\left|P_{a}-P_{b}\right|_{\text {max }}$, leading to lower $\epsilon_{B}$.

The Hybrid method generates almost an identical outcome as compared to the Liouville one, with a surprisingly good numerical accuracy. Similarly, a good agreement is achieved using the LZ as it reproduces well at a high MAS frequency even if it remains a bit off in the slow MAS regime. For a 3 spin system problem, the two simplified methods capture the spin physics. It highlights the accuracy of the methods at a fraction of the time cost, as simulations are 15 times faster for the Hybrid, and 20 times for the LZ approach.

\section{B. Accurate DNP field-sweep profile: the $b \mathrm{~T} b \mathrm{~K}$ example}

Thanks to the significant time-savings, one can now easily account for the presence of ${ }^{14} \mathrm{~N}$ spins in the binitroxides, which induce hyperfine EPR lineshifts. The resonant frequency of electron $i$ can be written as follows, assuming that the nuclear state of the ${ }^{14} \mathrm{~N}$ is $m_{i, I}$ and that the secular hyperfine coupling is $A_{i, z}^{I}$ :

$$
\omega_{i}\left(m_{I}\right)=g_{i} \beta B_{0}+m_{i, I} A_{i, z}^{I} .
$$

Note that this approach has already been implemented by other groups $[51,53,55]$ using other numerical codes with the goal to improve the field-sweep accuracy.

The importance of this feature is illustrated in Figure 5 for the $b \mathrm{~T} b \mathrm{~K}$ biradical case using a three spin system. Figure 5 (a) shows the simulated DNP field-sweep profile in the presence and absence of nitrogen hyperfine couplings. Their presences induce clear edges in the $g_{z}$ part of the profile, as well as a slight decrease of the positive maximum. In the end, this demonstrates that this new simulation tool is able to generate a field sweep profile in excellent agreement with previously published experimental data.[16]. This is of course of prerequisite for future work targeting in silico radical design.

In addition, Figure 5 (b) shows the effect of an increase of the $\mu \mathrm{w}$ irradiation strength $\omega_{1}$ on the DNP field-sweep profile: not only can it change the fieldsweep profile (e.g. features in the negative part of the field sweep) but it can also increase the enhancement factor at the optimal field position (about $4.696 \mathrm{~T}$ ). Remarkably, the ratio between the positive and negative part of the spectrum is also $\omega_{1}$ dependent. Note that simulations performed with multiple biradicals in a box gave the same normalized profile (not shown).

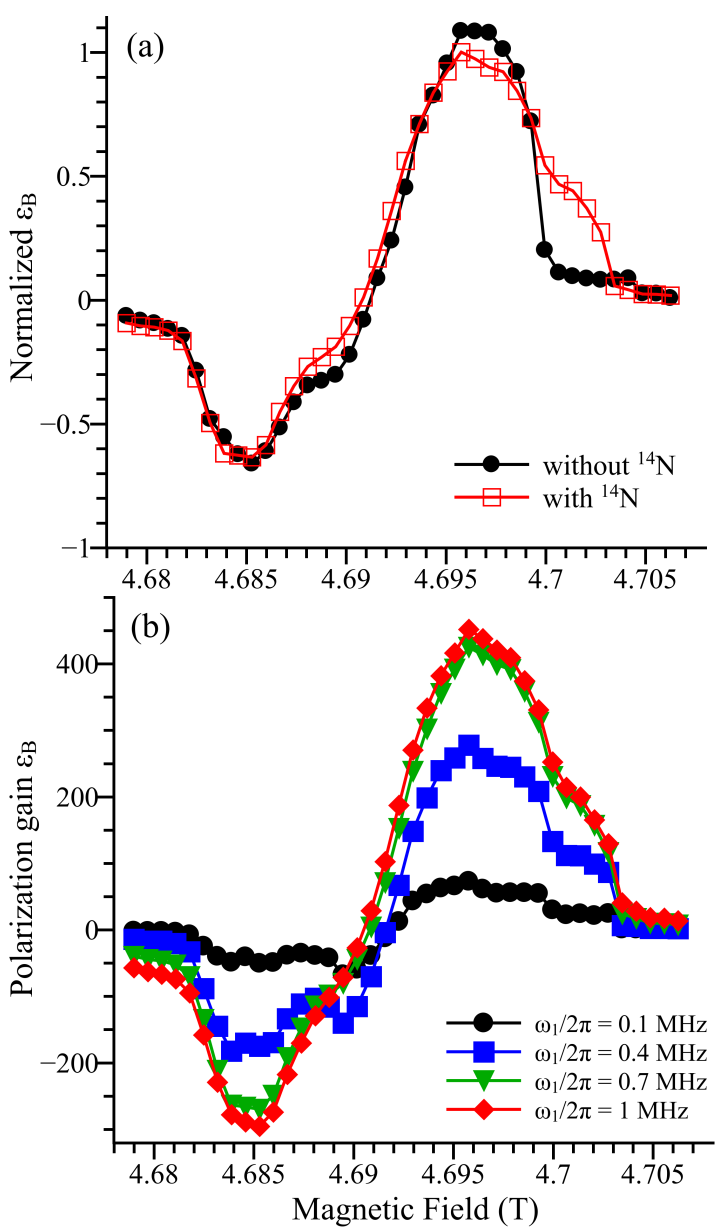

FIG. 5. (Three spins (e-e-n) simulations) Theoretical DNP Field sweep computed with the $b \mathrm{~T} b \mathrm{~K}$ geometry in the three spin system case. In figure (a), with (red) or without accounting the ${ }^{14} \mathrm{~N}$ hyperfine couplings. (b) Effect of the $\mu \mathrm{w}$ irradiation strength on the DNP Field sweep for 4 irradiation strengths (black circles $\omega_{1} / 2 \pi=0.1 \mathrm{MHz}$, blue squares $\omega_{1} / 2 \pi=0.4 \mathrm{MHz}$, green down-pointing triangles $\omega_{1} / 2 \pi=0.7 \mathrm{MHz}$, red diamonds $\omega_{1} / 2 \pi=1 \mathrm{MHz}$. For all the simulations, $T_{1}^{e}=0.3 \mathrm{~ms}, T_{2}^{e}=1 \mu \mathrm{s}, T_{1}^{n}=0.2 \mathrm{~s}$, $A_{1, a} / 2 \pi=1.5 \mathrm{MHz}, \nu_{\mu \mathrm{w}} / 2 \pi=131.725 \mathrm{GHz}, \nu_{r}=5 \mathrm{kHz}$ and $\omega_{1} / 2 \pi=0.7 \mathrm{MHz}$ for figure (a) where the $\mu \mathrm{w}$ irradiation strength has been used to obtain a good agreement with experimental data published in [16].

\section{Insight into multiple electron spin effects.}

Biradical concentration and $T_{1}^{e}$ effect: The effect of the biradical concentration was probed by computing the polarization gain $\epsilon_{B}$, the depolarization factor $\epsilon_{\text {Depo }}$, and the enhancement factor $\epsilon_{\text {On/Off }}$ following the methodology described in section II D 3 (see Figure 6). In all panels, the dotted line represents the simulations when the intermolecular interactions are zeroed. As clearly seen from Figure 6 , the intermolecular effect is not present for a $1 \mathrm{mM}$ concentration and 

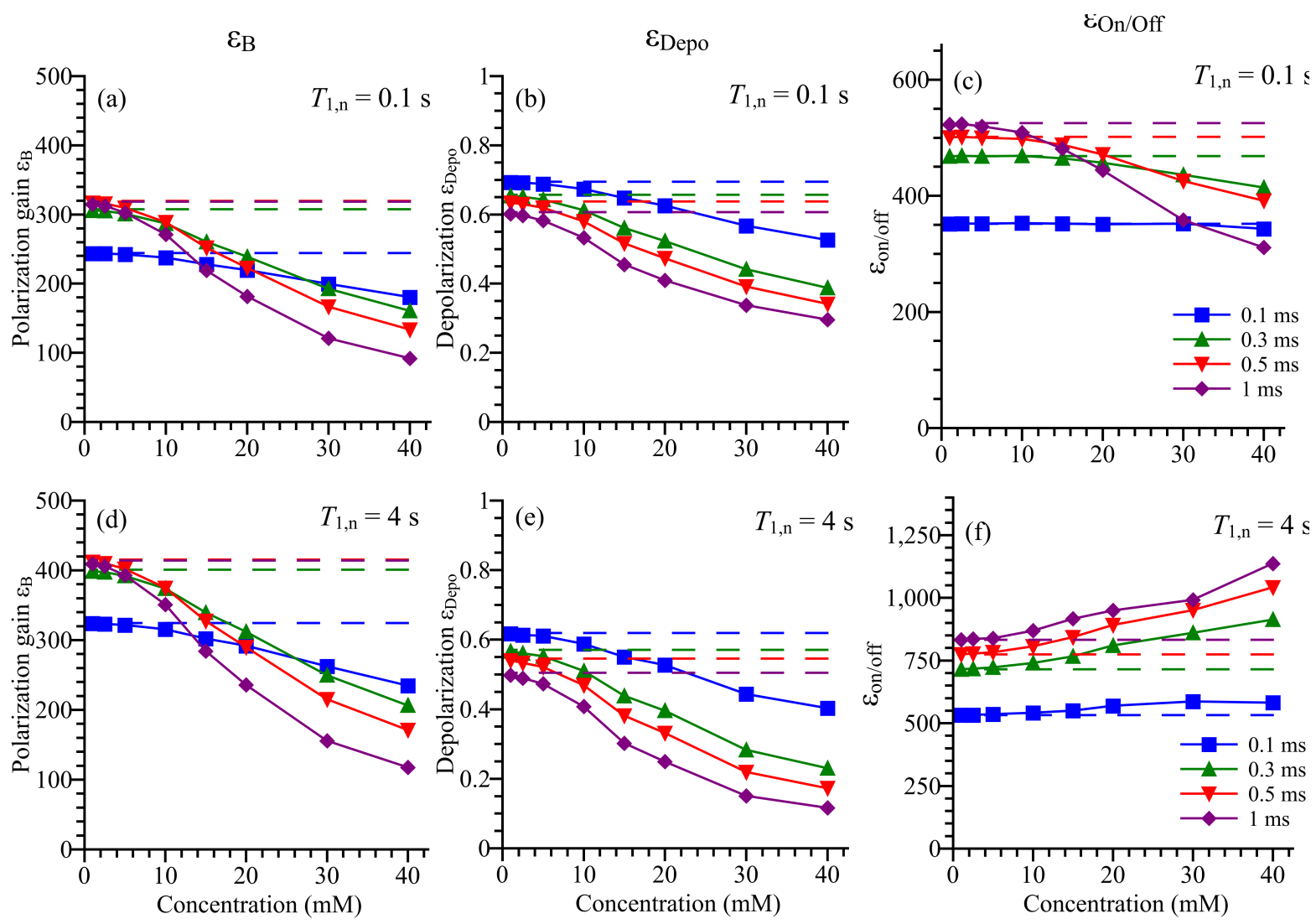

FIG. 6. (Box model simulation) Effect of the biradical concentration on the polarization gain $\epsilon_{B}$ (a and d), depolarization $\epsilon_{\text {Depo }}\left(\mathrm{b}\right.$ and $\mathrm{e}$ ) and $\epsilon_{\mathrm{On} / \mathrm{Off}}\left(\mathrm{c}\right.$ and $\mathrm{f}$ ) as a function of electron relaxation time $T_{1}^{e}=0.1 \mathrm{~ms}$ (blue squares), $0.3 \mathrm{~ms}$ (green uppointing triangles), $0.5 \mathrm{~ms}$ (red down pointing triangles), and $1 \mathrm{~ms}$ (purple diamonds). In all cases, $T_{2}^{e}=1 \mu \mathrm{s}, A_{1, a} / 2 \pi=1.5$ $\mathrm{MHz}, \omega_{\mu \mathrm{w}} / 2 \pi=263.45 \mathrm{GHz}, B_{0}=9.394 \mathrm{~T}, \nu_{r}=8 \mathrm{kHz}$ and $\omega_{1} / 2 \pi=0.7 \mathrm{MHz}$. For top part figures (a-c) $T_{1}^{n}=0.1 \mathrm{~s}$, and for bottom part figures (d-f) $T_{1}^{n}=4 \mathrm{~s}$. Simulations were performed by averaging 12 randomly distributed boxes containing 40 biradicals orientations (picked up among $144 \mathrm{ZCW}$ crystal orientations), and $d_{\min }=2 \mathrm{~nm}$. Dashed and solid lines represent respectively the isolated and interacting biradical case.

gradually increases with the biradical concentration.

Figure 6 (a) and (d) show that the polarization gain $\epsilon_{B}$ decreases with the concentration and that this effect is more pronounced for longer nuclear $T_{1}^{n}$ (0.1 versus $4 \mathrm{~s}$ ) and electronic (0.1 up to $1 \mathrm{~ms}) T_{1}^{e}$ relaxation times. Similarly $\epsilon_{\text {Depo }}$ (Figure 6 (b) and (e)) decreases (which means a greater depolarization effect) with increased concentrations and/or longer electron $T_{1}^{e}$. At this point it is worth noting that intermolecular effects can account for up to a factor 4 difference in terms of $\epsilon_{\text {Depo }}$ between isolated and coupled 3 spin systems at large biradical concentrations $(>10 \mathrm{mM})$. The presence of additional intermolecular electron-electron dipolar rotor events leads to a MAS-induced spectral diffusion, a mechanism that tends to equilibrate the electron polarization through the EPR line [53]. Such an effect is stronger at large electron concentration and for long $T_{1}^{e}$. Even with a simple "TOTAPOLlike" 3 spin system where only one nucleus collects the electron spin polarization difference, the MAS-induced
844 spectral diffusion clearly affects the electron polarization difference at steady state leading to a reduced hyperpolarization (i.e. smaller $\epsilon_{B}$ ) and a stronger depolarization effect (i.e. smaller $\epsilon_{\text {Depo }}$ ) $[50,53,54]$. Note that a longer $T_{1}^{n}$ for the local nuclei leads to a higher $\epsilon_{B}$ and lower $\epsilon_{\text {Depo }}$ in general.

On the contrary, the enhancement factor $\epsilon_{\mathrm{On} / \mathrm{Off}}$ commonly used, quoted, and relied upon in DNP studies has a very different behavior with respect to the electron concentration as can be seen in Figure 6 (c) and (f), and depends strongly on $T_{1}^{n}$ values. With the geometry and parameters considered here, $\epsilon_{\text {On } / O f f}$ decreases with concentration for short $T_{1}^{n}(0.1 \mathrm{~s})$ while it increases for longer values (4 s). Overall, the intermolecular couplings have a less pronounced effect on

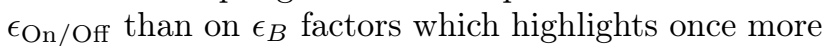
the limits of relying only on the former when comparing biradical efficiency or optimizing biradical geometry. Also, the bias introduced when using $\epsilon_{\mathrm{On} / \mathrm{Off}}$ is illustrated by the fact that values larger than the ratio 
of electron versus proton gyromagnetic ratio $(\sim 660)$ were easily computed with this reduced 3 spin system.

All in all, these simulations indicate the interest of using rather low biradical concentrations $(<10 \mathrm{mM})$ and relatively long electron relaxation times (up to $T_{1}^{e} \leq 0.5 \mathrm{~ms}$ ). At this point, it is important to note that the results discussed here were obtained for a given biradical geometry (close to TOTAPOL) and a selected set of nuclear/electron relaxation times, and neglecting the role of the bulk nuclei. We believe that such simulations give a good qualitative picture of the CE MAS-DNP mechanism but that different results could be obtained with other input parameters, and when the bulk nuclei are taken into account the behavior of the concentration may be changed.

Main magnetic field dependency Figure 7 presents similar simulations as in Figure 6 but exploring the effect of the magnetic field (corresponding to 200 to 800 $\mathrm{MHz}{ }^{1} \mathrm{H}$ Larmor frequencies) on the CE MAS-DNP efficiency. The spin system is the same as in the previous section and the biradical concentration fixed to 15 mM. Overall the polarization gain $\epsilon_{B}$ (panels (a) and (d)) decreases with increasing magnetic field and this effect is more pronounced for short nuclear relaxation times $(0.1$ versus $4 \mathrm{~s})$. In-line with the previous section, we observe that the polarization gain $\epsilon_{B}$ is hardly affected by the presence of MAS induced spectral diffusion at short electron relaxation times $T_{1}^{e}=0.1 \mathrm{~ms}$ (blue squares) but strongly decreased at longer times $\left(T_{1}^{e}>0.3 \mathrm{~ms}\right)$. For instance, for $T_{1}^{e}=1 \mathrm{~ms}, \epsilon_{B}$ is reduced from 380 to 200 when taking into account intermolecular interactions (15 mM concentration) at $B_{0}=4.7 \mathrm{~T}$. Figure 7 also illustrates that long $T_{1}^{e}$ are especially preferred at high magnetic fields $(>15-20$ T) but not necessarily for lower field studies. Once more these simulations illustrate the importance of relying on the polarization gain $\epsilon_{B}$ and not the $\epsilon_{\text {On/Off }}$ enhancement factor since they clearly give two different qualitative pictures: longer $T_{1}^{e}$ always provide higher $\epsilon_{\text {On/Off }}$ values whereas the situation is more complex in terms of real polarization gain. Notably, long $T_{1}^{e}(1$ $\mathrm{ms}$ ) at low field yield a lower polarization gain. The discrepancy between $\epsilon_{B}$ and $\epsilon_{\text {On/Off }}$ can be explained by looking at the depolarization factor $\epsilon_{\text {Depo }}$. It is worth noting that a significant part of the depolarization comes from intermolecular effect and that this contribution gets smaller at higher fields. This is actually consistent with recent experimental findings[50]. The depolarization effect (intra and intermolecular contribution) is larger at low magnetic fields and/or for long $T_{1}^{e}$ values.

The theoretical results presented here are inline with the trends previously observed experimentally and theoretically for $\epsilon_{\mathrm{On} / \mathrm{Off}}, \epsilon_{\mathrm{Depo}}$, and $\epsilon_{B}[50,54]$. We must of course emphasize that the results presented here were obtained for 40 biradicals with a TOTAPOL-like As the exact values are not known, we assumed that the relaxation time of nucleus $i$ is given by

$$
\frac{1}{T_{1, i}^{n}}=\frac{1}{T_{1,1}^{n}}\left(\frac{A_{a, 1}}{A_{a, i}}\right)^{2}+\frac{1}{T_{1, \text { Bulk }}^{n}}
$$

where $T_{1,1}^{n}$ is the relaxation time of the closest nuclei

geometry in a box with a set of interaction and relaxation parameters. Therefore these results should not straightforwardly be compared with experimental observations from samples that contain different types of biradicals and differ in their nuclear conformations.

\section{Accounting for multiple nuclear spins}

In this section we investigate the polarization of a large set of protons in a two-electron system as deibed in section II D 2. The spin system considered as section consists of isolated biradicals coupled to a set of 400 protons. These protons are divided in trons and bulk protons coupled to the Local/ENDOR protons that exchange their polarization via spin diffusion. A key feature of the model is that one can easily introduce non-uniform nuclear relaxation times among the local protons in order to account for the fact that they are not all located at the same distance from the electrons. More precisely the model presented below assumes that the electron spin-flips induce through the do-secular hyperfine coupling $\left(A_{a, n}^{ \pm}\right)$a field fluctuation at the proton position that leads to a nuclear relaxation mechanism [69] given by $T_{1}^{n}$ :

$$
\frac{1}{T_{1}^{n}} \propto\left|A_{a, n}^{ \pm}\right|^{2}(S(S+1)) \frac{\tau}{1+\omega_{n}^{2} \tau^{2}}
$$

where $\tau$ is an electron spin-flip correlation time that can be close to either $T_{1}^{e}$ or $T_{2}^{e}$ depending on the concentration [69]. The nuclear relaxation is then proportional to the square of the hyperfine coupling $\left(T_{1}^{n} \propto\right.$ $\left.\left|A_{a, n}\right|^{-2} \propto r^{6}\right)$. Nonetheless, the biradicals are not the only source of relaxation. Indeed, in an undoped frozen solution the relaxation time has still a finite value (of 30 - $80 \mathrm{~s}[19,30,50])$, which originates from other relaxation mechanisms such as proton-proton dipolar relaxation or dissolved paramagnetic oxygen. It is reasonable to assume that these additional mechanisms are responsible for the relaxation of the bulk nuclei, while the local protons relax under the influence of the biradicals. The nuclear relaxation rates can then be written as the sum of the two contributions

$$
\frac{1}{T_{1}^{n}}=\frac{1}{T_{1, a-n}^{n}}+\frac{1}{T_{1, \mathrm{Bulk}}^{n}}
$$



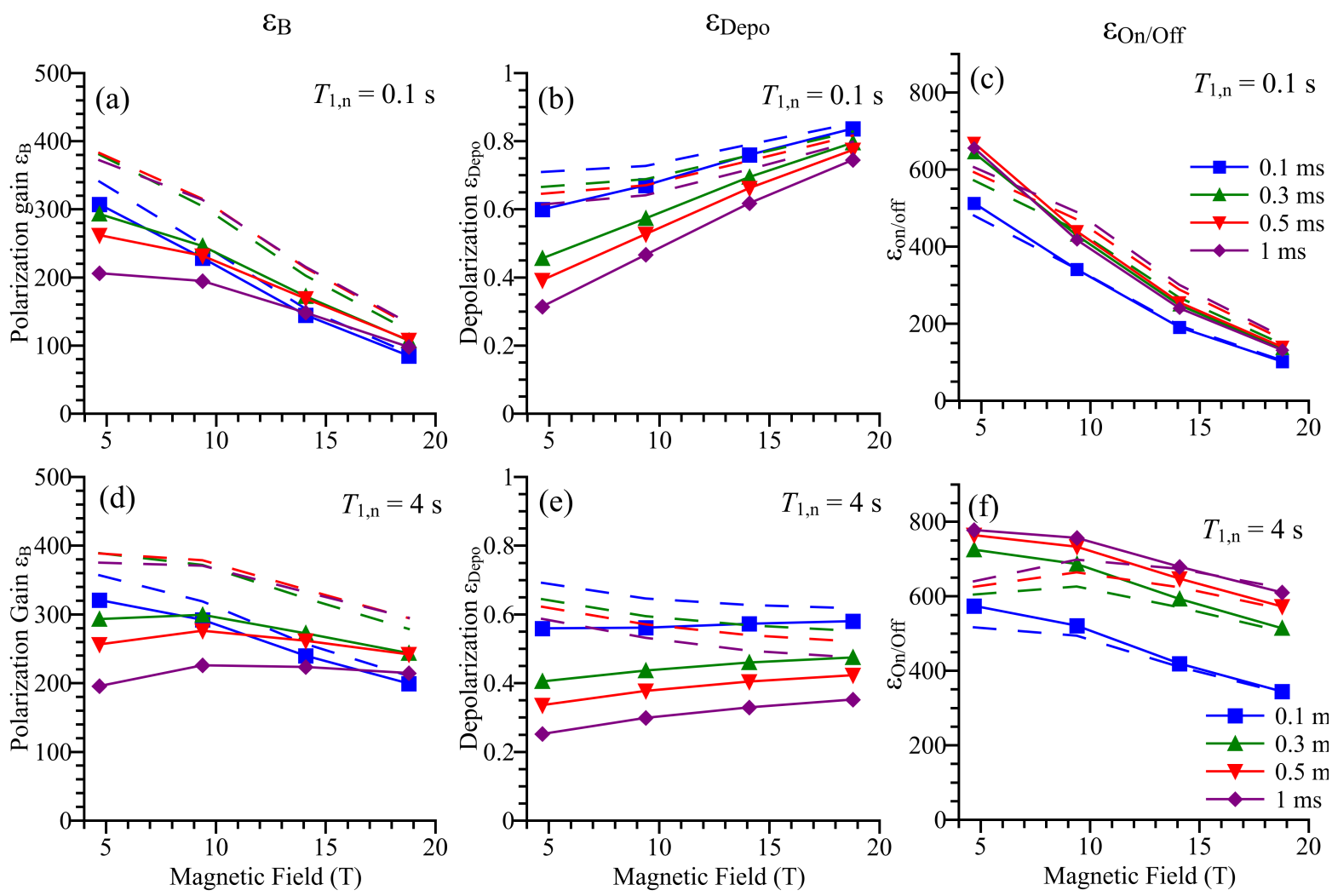

FIG. 7. (Box model simulation) Dependence of the polarization gain $\epsilon_{B}$ (a and d), depolarization $\epsilon_{\text {Depo }}(\mathrm{b}$ and e) and $\epsilon_{\mathrm{On} / \mathrm{Off}}(\mathrm{c}$ and $\mathrm{f})$ as a function of the main magnetic field and electron relaxation time, $T_{1}^{e}=0.1 \mathrm{~ms}$ (blue squares), $0.3 \mathrm{~ms}$ (green up-pointing triangles), $0.5 \mathrm{~ms}$ (red down pointing triangles), and $1 \mathrm{~ms}$ (purple diamonds). The biradical structure used corresponds to TOTAPOL, $T_{2}^{e}=1 \mu \mathrm{s}, A_{1, a} / 2 \pi=1.5 \mathrm{MHz}, \omega_{\mu \mathrm{w}} / 2 \pi=263.45 \mathrm{GHz}, B_{0}=9.394 \mathrm{~T}, \nu_{r}=8 \mathrm{kHz}$ and $\omega_{1} / 2 \pi=0.7 \mathrm{MHz}$. Top figures (a-c) $T_{1}^{n}=0.1 \mathrm{~s}$, and bottom figures (d-f) $T_{1}^{n}=4 \mathrm{~s}$. Simulations with the Hybrid approach have been performed by averaging 10 randomly distributed boxes containing 40 biradicals orientations (picked up among 144 ZCW crystal orientations) with a concentration of $15 \mathrm{mM}$, and $d_{\min }=2 \mathrm{~nm}$. Dotted and solid lines represent respectively the isolated and interacting biradical case.

and $T_{1, \mathrm{Bulk}}^{n}$ the bulk nuclear relaxation times. This arbitrary choice allows to have a continuous set of $T_{1}^{n}$ that that can reach very short values. The calculation presented in Figure 8 was performed with a spin system made of $N_{L}=60$ local nuclei spread over a 4 layers cone. Details about the spin system and the calculation can be found in section II D 2. The typical simulation time required for a single orientation and 400 nuclei ( 60 local, 340 bulk) is about $200 \mathrm{~s}$, about 20 times faster than a full Liouville calculation with only 3 nuclear spins. The simulated build-up times $T_{\mathrm{B}}$ were obtained after fitting the average build-up curve of the bulk nuclei with a single exponential.

Effect of the nuclear dipolar rotor events: absence of a spin diffusion barrier Figure 8 presents the polarization build-up $\epsilon_{B}(t)$ of the local nuclei in the absence (a) and presence (b) of the nuclear-dipolar couplings. The spin system is described in section II D. 2. In Figure 8 (a), the build-ups can be classified into four groups, each of them corresponding to one of the four layers of local nuclei. The two closest nuclei (black and blue curves) have a fast build up and reach significant polarization while the other nuclei tend to have a slower build-up time and reach a lower polarization gain. The mean polarization build-up is represented by the thick blue line. It corresponds to a stretched exponential shape and only reaches up to a polarization gain of 5. Note that in this simulation, bulk protons were not considered. In Figure 8 (b), only 3 buildups can be observed: the two closest nuclei can still be differentiated ((black and blue lines) in terms of $\epsilon_{B}$, whereas the rest of the local nuclei now have the same polarization build-up. The first two nuclei reach a higher polarization compared to the other local nuclei, and have a build-up that is bi-exponential, with a fast and a much slower component. The other local nuclei have a common build-up time equal to the slow component of the first two nuclei. 


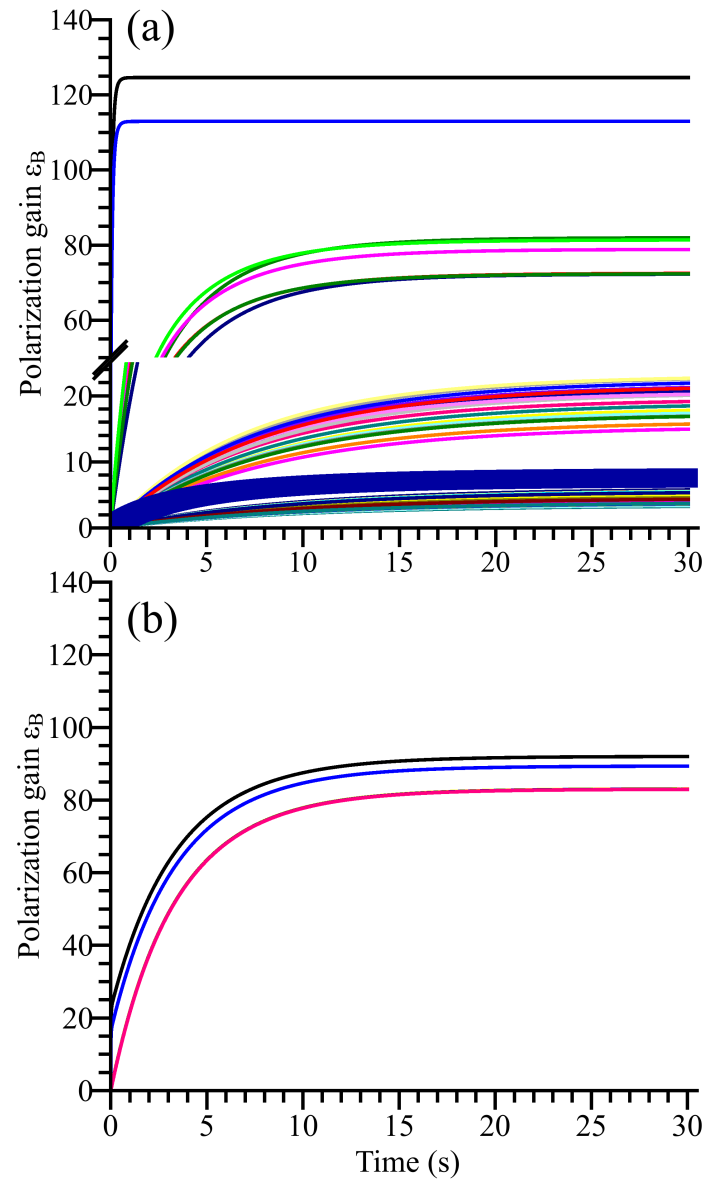

FIG. 8. (Bulk model simulation) Polarization build-up $\epsilon_{B}{ }^{1028}$ for local nuclei without (a), and (b) with nuclear-dipolar 1029 rotor events (i.e. nuclear spin-diffusion). Black curve cor- 1030 responds to the first proton, blue curve to the second pro- 1031 ton, and the following to the other shells. In (a), the ${ }_{1032}$ thick blue curve represents the mean polarization buildup. Simulation performed for TOTAPOL geometry with $\omega_{1} / 2 \pi=0.85 \mathrm{MHz}, T_{1}^{e}=0.3 \mathrm{~ms}, T_{2}^{e}=1 \mu \mathrm{s}, A_{1, a} / 2 \pi=3$ $\mathrm{MHz}, \omega_{\mu \mathrm{w}} / 2 \pi=263.45 \mathrm{GHz}, B_{0}=9.394 \mathrm{~T}, \nu_{r}=8 \mathrm{kHz}$. In (a), the bulk relaxation time was $T_{1, \mathrm{Bulk}}^{n}=10 \mathrm{~s}$, the closest proton relaxation time was $T_{1,1}^{n}=0.15 \mathrm{~s}$.

When the nuclear-nuclear dipolar couplings are absent, Figure 8 (a) shows that the polarization among the nuclei presents a steep gradient, that the mean polarization only reaches a small value, and that long- ${ }^{1043}$ distant nuclei are hardly polarized. In addition, the ${ }^{1044}$ mean polarization curve does not appear to be a simple ${ }^{1045}$ exponential similar to what is observed in certain DNP 1046 experiments where spin diffusion is inefficient [77-79]. ${ }^{1047}$ In contrast, in presence of nuclear-dipolar rotor events, ${ }^{1048}$ even inefficient ones, the polarization is homogeneous ${ }^{1049}$ amongst the nuclei. The polarization of the two closest 1050 nuclei is heavily reduced and tends to be much closer ${ }_{1051}$ to the polarization of the rest of the local nuclei. The ${ }_{1052}$ mean polarization is higher than in Figure 8 (a). The 1053

tino

fact, however, that the proton polarization tends to be equalized highlights the lack of a "spin diffusion barrier". Figure 8 (b) indicates that the first and second nuclei layers, are the polarization "feeding source" of all nuclei as the mean polarization of the local nuclei is higher in case (b) than in (a). The presence of nuclear spin diffusion (via the nuclear dipolar rotor events) allows more than just averaging the polarization, it allows the high polarization to flow from the very close protons to the distant ones. This confirms preliminary simulations [54] and reveals that even the very close protons (those on the biradical) can be active members of the DNP process. Note that this last observation can be inferred from experimental results on deuterated biradicals [45, 47, 48], but was lacking theoretical support up to now. It is also interesting to note that increasing the number of protons to more realistic values allows predicting polarization gains and build-up times much closer to the experimental values.

Effect of the $T_{1,1}^{n}$ and $T_{1, B u l k}^{n}$ The impact of the nuclear relaxation times on the mean $\epsilon_{B}$ and mean polarization time $T_{\mathrm{B}}$ was probed. The evolution is plotted in Figure 9 (a) for different $T_{1,1}^{n}$ and in Figure 9 (b) for different $T_{1, \text { Bulk }}^{n}$. In (a) $T_{1, \text { Bulk }}^{n}$ is constant, $T_{1,1}^{n}$ is varied and the relaxation times of local nuclei obey Equation 20. $\epsilon_{B}$ is larger with increased relaxation time $T_{1,1}^{n}$. Remarkably it appears that the polarization time $T_{\mathrm{B}}$ and the final enhancement $\epsilon_{B}$ vary linearly. This is represented in the insert of Figure 9 (a) (black curve). A similar behavior is observed in Figure 9 (b) where $T_{1,1}^{n}$ was kept constant while $T_{1, \text { Bulk }}^{n}$ is varied. Here as well, when the bulk relaxation gets longer the build up times also become longer and the polarizations reach higher values. On the range tested, the relationship between $\epsilon_{B}$ and $T_{\mathrm{B}}$ seems to obey a very similar law (see blue curve in the insert of figure (a)). This linear behavior is also observed for larger electron-electron dipolar interactions (See SI section C.).

In conclusion we observe that for a given geometry (electrons and protons) the DNP efficiency, i.e. the polarization gain and the polarization buildup time, is directly influenced by the local protons' relaxation properties as long as the bulk proton $T_{1}^{n}$ (obtained in absence of radicals) is longer than the build-up times $T_{\mathrm{B}}$.

Effect of the number of nuclei Figure 10 reports the effect of the number of bulk protons $N_{\text {Bulk }}$ on the DNP efficiency (polarization gain $\epsilon_{B}$ and build-up time $T_{\mathrm{B}}$ ). When $N_{\text {Bulk }}$ increases, the build-up time gets longer while the polarization gain $\epsilon_{B}$ is decreased. This observation (that can be intuitively assessed) is valid for the two $T_{1, \text { Bulk }}^{n}$ values tested here $(60$ and $5 \mathrm{~s})$ and more pronounced for the shorter $T_{1, \mathrm{Bulk}}^{n}$ value.

Effect of the electron-electron dipolar and hyperfine interaction The electron-electron dipolar coupling $D_{a, b}$ and the external magnetic field were var- 

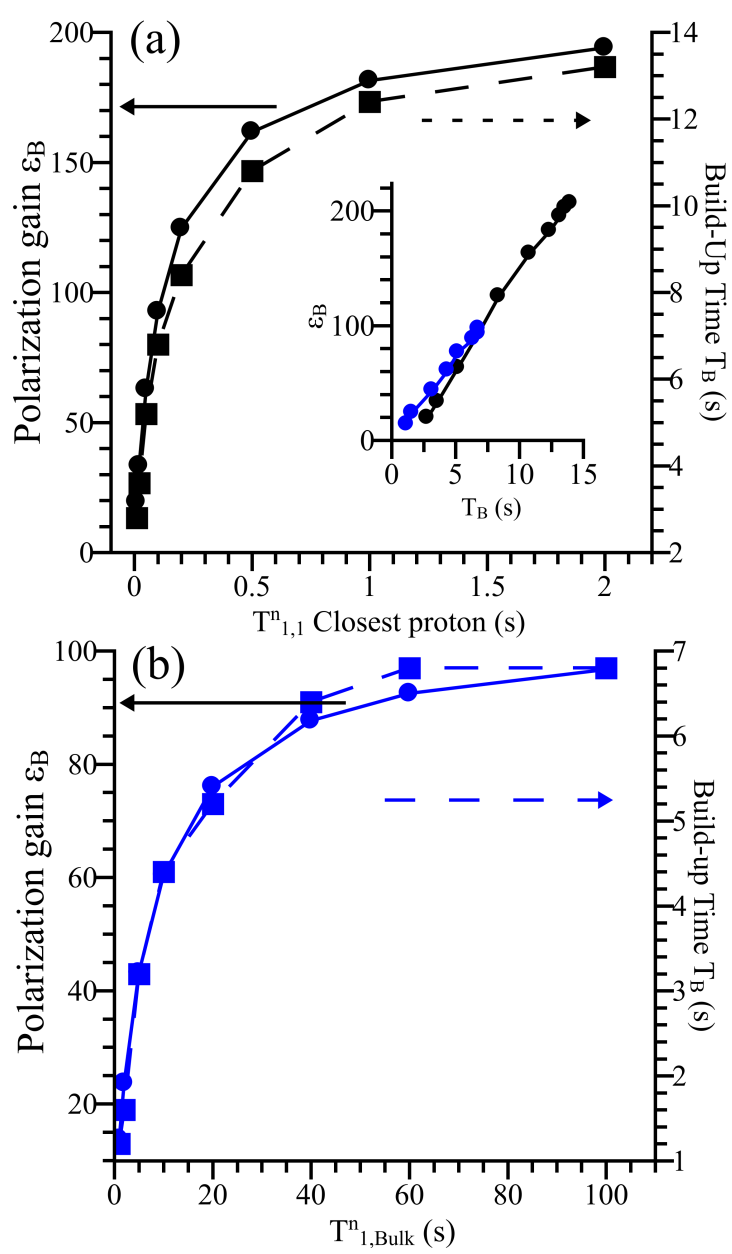

FIG. 9. (Bulk model simulation) (a) Effect of $T_{1,1}^{n}$ on the polarization gain $\epsilon_{B}$ (black curve, left axis) and build-up time $T_{\mathrm{B}}$ (black dashed curve, right axis). (b) Effect of $T_{1, \text { Bulk }}^{n}$ on the polarization gain $\epsilon_{B}$ (blue curve, left axis) ${ }^{1069}$ and build-up time $T_{\mathrm{BU}}$ (blue dotted curve, right axis) Cal- 1070 culations were performed with the spin system described in $\mathbf{1 0 7 1}$ section II D 2 using a "TOTAPOL-like" geometry and the 1072 following input parameters: $\omega_{1} / 2 \pi=0.85 \mathrm{MHz}, T_{1}^{e}=0.3{ }_{1073}$ $\mathrm{ms}, T_{2}^{e}=1 \mu \mathrm{s}, A_{1, a} / 2 \pi=3 \mathrm{MHz}, \omega_{\mu \mathrm{w}} / 2 \pi=263.45 \mathrm{GHz}$, $B_{0}=9.394 \mathrm{~T}, \nu_{r}=8 \mathrm{kHz}$. In (a), the bulk relaxation time was $T_{1, \text { Bulk }}^{n}=60 \mathrm{~s}$, while in (b) $T_{1,1}^{n}=0.1 \mathrm{~s}$. Insert in (a) represents $\epsilon_{B}$ as a function of $T_{\mathrm{B}}$ in both cases.

ied and the simulations are reported in Figure 11. As 1080 in the 3 spins system case, the increase in the mag- 1081 netic field leads to a significant decrease of the po- ${ }_{1082}$ larization gain $\epsilon_{B}$ while increasing the build-up times 1083 $T_{\mathrm{B}}$. The calculations are inline with experimental ob- 1084 servations reported for the two water soluble biradi- 1085 cals TOTAPOL and AMUPol [21, 50]. The polariza- 1086 tion build-up times increase with the magnetic field for 1087 both biradicals, whereas the higher dipolar coupling in 1088 AMUPol explains why it polarizes better and faster 1089 than TOTAPOL.

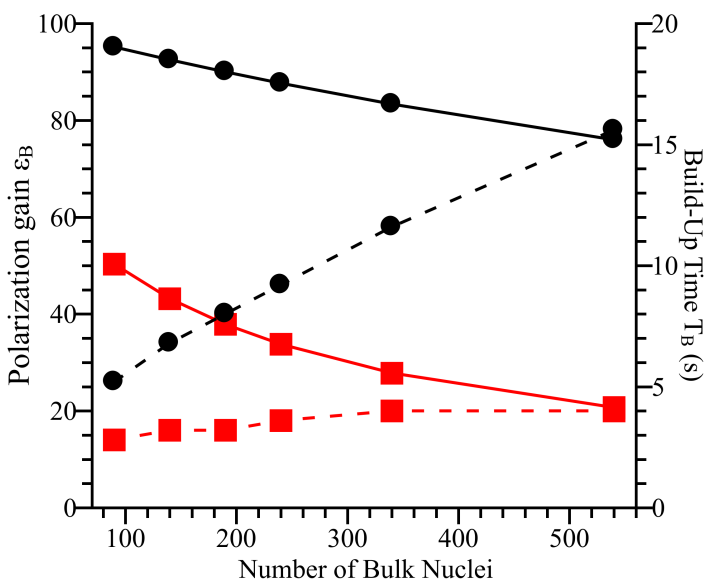

FIG. 10. (Bulk model simulation) Effect of the number of bulk nuclei $N_{\text {Bulk }}$ on the final $\epsilon_{B}$ (black curve, right axis) and build-up time $T_{\mathrm{B}}$ (black dashed curve, right axis) for $T_{1, \text { Bulk }}^{n}=60 \mathrm{~s}$, and $\epsilon_{B}$ (red curve, left axis) and buildup time $T_{\mathrm{B}}$ (red dashed curve, right axis) for $T_{1, \text { Bulk }}^{n}=5$ s. Simulations performed for TOTAPOL geometry with $\omega_{1} / 2 \pi=0.85 \mathrm{MHz}, T_{1}^{e}=0.3 \mathrm{~ms}, T_{2}^{e}=1 \mu \mathrm{s}, A_{1, a} / 2 \pi=3$ $\mathrm{MHz}, \omega_{\mu \mathrm{w}} / 2 \pi=263.45 \mathrm{GHz}, B_{0}=9.394 \mathrm{~T}, \nu_{r}=8 \mathrm{kHz}$, the closest proton relaxation time was $T_{1,1}^{n}=0.1 \mathrm{~s}$.

Figure 12 shows the dependence of both the polarization gain $\epsilon_{B}$ and the build-up time $T_{\mathrm{B}}$ when varying the hyperfine couplings to the local protons. In the simulation presented here, all the protons are moved together as a whole and the effect on the DNP efficiency is plotted against the hyperfine coupling to the closest proton. Note that for each cases, all hyperfine couplings and $T_{1, i}^{n}$ relaxation times are changed according to their distance to electron a and the formulae given in equation 20 respectively. Figure 12 presents two sets of curves for which $T_{1,1}^{n}=0.1$ or $0.5 \mathrm{~s}$ respectively for a hyperfine coupling of $A_{a, 1} / 2 \pi=3 \mathrm{MHz}$. A stronger hyperfine coupling generates a higher polarization gain and a faster build-up time. As discussed in a previous paragraph, longer nuclear relaxation times of the closest protons $T_{1,1}^{n}$ induces larger polarization gain and longer build-up time. It is interesting to note that a given polarization gain can be reached either with a small hyperfine coupling and a long nuclear relaxation time $T_{1,1}^{n}$ of the closest nuclei or with a stronger hyperfine and a shorter nuclear relaxation time. It is also worth noting that reasonably short build-up times are only obtained when the strongest hyperfine coupling are of the order of $1 \mathrm{MHz}$. This again supports the strong role of nearby nuclei in the MAS-DNP process. 


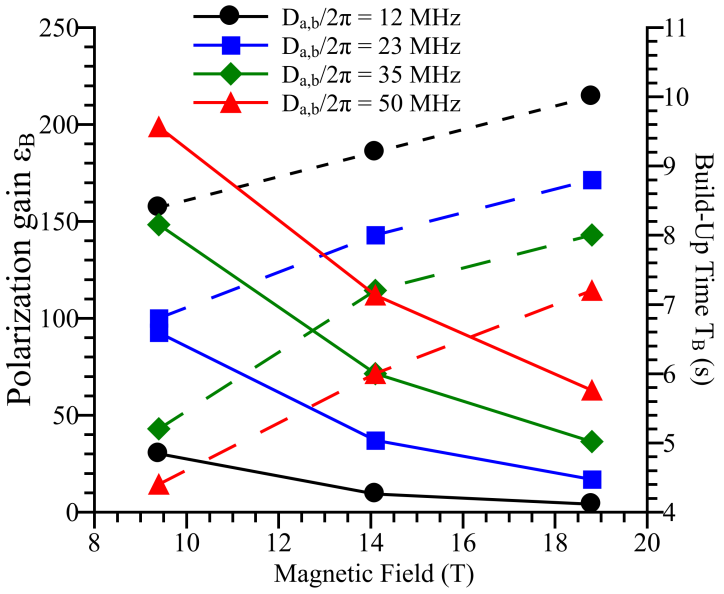

FIG. 11. (Bulk model simulation) Effect of main magnetic field on the mean $\epsilon_{B}$ (full curve, left axis) and build-up time $T_{\mathrm{B}}$ (dashed curve, right axis), for different electron-electron $D_{a, b} / 2 \pi=12 \mathrm{MHz}$ (black circles), $23 \mathrm{MHz}$, (blue squares), $35 \mathrm{MHz}$ (green diamonds) and $50 \mathrm{MHz}$ (red triangles) performed for TOTAPOL geometry with $\omega_{1} / 2 \pi=0.85 \mathrm{MHz}$, $T_{1}^{e}=0.3 \mathrm{~ms}, T_{2}^{e}=1 \mu \mathrm{s}, T_{1}^{n}=0.2 \mathrm{~s}, A_{1, a} / 2 \pi=3 \mathrm{MHz}$, $\nu_{r}=8 \mathrm{kHz}$. The bulk relaxation times was $T_{1, \text { Bulk }}^{n}=60 \mathrm{~s}$, the closest proton relaxation time was $T_{1,1}^{n}=0.1 \mathrm{~s}$.

\section{CONCLUSION AND PERSPECTIVES}

In this work, we introduce a new model to compute efficiently Cross-Effect and Solid-Effect MASDNP mechanisms with the aim to build a predictive tool that can be used not only to understand polariza- 1119 tion transfer mechanisms but also to design efficient 1120 polarizing agents in the future. The formalism is a ${ }^{1121}$ combination of Bloch-type derivations and LandauZener approximations and is in excellent agreement ${ }_{1122}$ with full Liouville calculations. Overall, we provide ${ }_{1123}$ simulations of the DNP efficiency, in terms of polariza- ${ }_{1124}$ tion gains $\epsilon_{B}$ and "enhancement factors" $\epsilon_{\text {On/Off }}$ but 1125 also build-up times, for various key parameters. As ${ }_{1126}$ demonstrated in this work, and thanks to the signif- ${ }_{1227}$ icant time-savings afforded by the approach, one can ${ }_{1128}$ easily scan through multiple parameters and disentan- 1129 gle their mutual influences. In addition, the simula- 1130 tion code is able to handle multiple electrons and pro- ${ }_{1131}$ tons, which allows probing electron concentration ef- ${ }_{1132}$ fects as well as fully revealing the interplay between 1133 nuclear dipolar couplings, hyperfine couplings, nuclear ${ }_{1134}$ relaxation times, and the important role of the nearby 1135 nuclei. It was possible to easily account for the ${ }^{14} \mathrm{~N}{ }_{1136}$ hyperfine couplings so as to provide $b \mathrm{~T} b \mathrm{~K}$ field-sweep ${ }_{1137}$ profiles in very good agreement with experiments. Finally simulations performed with multiple nuclei revealed the impact of the close nuclei on the DNP process, and also allowed discussing the absence of spindiffusion barrier, and the difference between the appar-

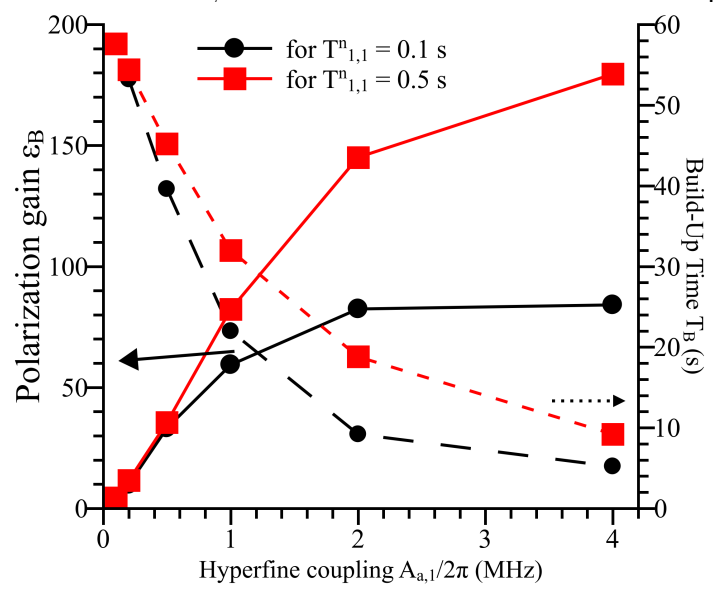

FIG. 12. (Bulk model simulation) Effect of the hyperfine coupling to the closest nuclei on the mean $\epsilon_{B}$ (full curve, left axis) and build-up time $T_{\mathrm{B}}$ (dashed curve, right axis), for two different cases, for which the closest nuclear relaxation time was first calibrated to $T_{1,1}^{n}=0.1 \mathrm{~s}$ (black) and $0.5 \mathrm{~s}$ (red) for $A_{a, 1} / 2 \pi=3 \mathrm{MHz}$. The simulations were performed for a TOTAPOL geometry with $\omega_{1} / 2 \pi=0.85$ $\mathrm{MHz}, T_{1}^{e}=0.3 \mathrm{~ms}, T_{2}^{e}=1 \mu \mathrm{s}, \omega_{\mu \mathrm{w}} / 2 \pi=263.45 \mathrm{GHz}$, $B_{0}=9.394 \mathrm{~T}, \nu_{r}=8 \mathrm{kHz}$, and, the bulk relaxation times was $T_{1, \text { Bulk }}^{n}=60 \mathrm{~s}$. Details can be found in the text.

ent DNP buildup time $T_{\mathrm{B}}$ and the local/Bulk nuclear relaxation times..

\section{ACKNOWLEDGEMENTS}

Daniel Lee, Sabine Hediger and Yonatan Hovav are acknowledged for insightful discussions throughout the course of the work. Adam Smith, Katharina Märker, Ildefonso Marin-Montesinos and Sachin Chaudari for interesting feedbacks and comments on the manuscript. F.M.V. acknowledges the COST Action TD 1103, STSM program. This work was supported in part by the French National Research Agency through the "programme blanc" (ANR-12-BS08-001601), the Labex ARCANE (ANR-11-LABX-0003-01) and the European Research Council (ERC-CoG-2015, No. 682895). Funding from the RTB is acknowledged. This research was made possible in part by the historic generosity of the Harold Perlman Family. S.V. holds the Joseph and Marian Robbins Professorial Chair in Chemistry.
[1] A. W. Overhauser, Phys. Rev. 92, 411 (1953).
[2] T. R. Carver and C. P. Slichter, Phys. Rev. 92, 212 (1953). 
[3] L. R. Becerra, G. J. Gerfen, R. J. Temkin, D. J. Singel, 1203 and R. G. Griffin, Phys. Rev. Lett. 71, 3561 (1993). $\quad 1204$

[4] D. A. Hall, Science (80-. ). 276, 930 (1997).

[5] T. Maly, G. T. Debelouchina, V. S. Bajaj, K.-N. N. Hu, ${ }_{1206}$ C.-G. C.-G. Joo, M. L. Mak-Jurkauskas, J. R. Sirigiri, 1207 P. C. A. Van Der Wel, J. Herzfeld, R. J. Temkin, and 1208 R. G. Griffin, J. Chem. Phys. 128, 052211 (2008). 1209

[6] A. B. Barnes, G. De Paëpe, P. C. A. Van Der Wel, 1210 K.-N. N. Hu, C. G. Joo, V. S. Bajaj, M. L. Mak- 1211 Jurkauskas, J. R. Sirigiri, J. Herzfeld, R. J. Temkin, 1212 and R. G. Griffin, Appl. Magn. Reson. 34, 237 (2008). 1213

[7] M. Rosay, L. Tometich, S. Pawsey, R. Bader, 1214 R. Schauwecker, M. Blank, P. M. Borchard, S. R. 1215 Cauffman, K. L. Felch, R. T. Weber, R. J. Temkin, 1216 R. G. Griffin, and W. E. Maas, Phys. Chem. Chem. 1217 Phys. 12, 5850 (2010).

[8] Q. Z. Ni, E. Daviso, T. V. Can, E. Markhasin, S. K. 1219 Jawla, T. M. Swager, R. J. Temkin, J. Herzfeld, and 1220 R. G. Griffin, Acc Chem Res 46, 1304250100250081221 (2013).

9] U. Akbey, W. T. Franks, A. Linden, M. OrwickRydmark, S. Lange, and H. Oschkinat, in Top. Curr. ${ }_{122}$ Chem., Vol. 338 (2013) pp. 181-228.

[10] A. J. Rossini, A. Zagdoun, M. Lelli, A. Lesage, C. Cop- ${ }_{1226}$ eret, and L. Emsley, Acc. Chem. Res. 46, 1942 (2013). 1227

[11] D. Lee, S. Hediger, and G. De Paëpe, Solid State Nucl. ${ }_{1228}$ Magn. Reson. 66-67, 6 (2015).

12] T. Kobayashi, F. A. Perras, I. I. Slowing, A. D. Sadow, 1230 and M. Pruski, ACS Catal. 5, 7055 (2015). 1231

[13] A. N. Smith and J. R. Long, Anal. Chem. 88, 1221232 (2016).

[14] K.-N. N. Hu, H.-h. Yu, T. M. Swager, and R. G. ${ }_{1234}^{1233}$ Griffin, J. Am. Chem. Soc. 126, 10844 (2004). 1235

[15] C. Song, K.-N. N. Hu, C.-G. Joo, T. M. Swager, and 1236 R. G. Griffin, J. Am. Chem. Soc. 128, 11385 (2006). ${ }_{1237}$

[16] Y. Matsuki, T. Maly, O. Ouari, H. Karoui, F. Le 1238 Moigne, E. Rizzato, S. Lyubenova, J. Herzfeld, T. F. 1239 Prisner, P. Tordo, and R. G. Griffin, Angew. Chem. 1240 Int. Ed. Engl. 48, 4996 (2009).

[17] A. Lesage, M. Lelli, D. Gajan, M. A. Caporini, 1242 V. Vitzthum, P. Miéville, J. Alauzun, A. Roussey, 1243 C. Thieuleux, A. Mehdi, G. Bodenhausen, C. Copéret, 1244 and L. Emsley, J. Am. Chem. Soc. 132, 15459 (2010). 1245

[18] E. L. Dane, B. Corzilius, E. Rizzato, P. Stocker, 1246 T. Maly, A. A. Smith, R. G. Griffin, O. Ouari, 1247 P. Tordo, and T. M. Swager, J. Org. Chem. 77, 17891248 (2012).

[19] O. Haze, B. Corzilius, A. A. Smith, R. G. Griffin, and 1250 T. M. Swager, J. Am. Chem. Soc. , 1 (2012). 1251

[20] M. K. Kiesewetter, B. Corzilius, A. A. Smith, R. G. 1252 Griffin, and T. M. Swager, J. Am. Chem. Soc. 134, 1253 4537 (2012).

[21] C. Sauvee, M. Rosay, G. Casano, F. Aussenac, R. T. ${ }_{1255}$ Weber, O. Ouari, and P. Tordo, Angew. Chemie Int. 1256 Ed. 52, 10858 (2013).

[22] A. Zagdoun, G. Casano, O. Ouari, G. Lapadula, A. J. Rossini, M. Lelli, M. Baffert, D. Gajan, L. Veyre, 1259 W. E. Maas, M. Rosay, R. T. Weber, C. Thieuleux, 1260 C. Coperet, A. Lesage, P. Tordo, and L. Emsley, J. 1261 Am. Chem. Soc. 134, 2284 (2012).

[23] A. Zagdoun, G. Casano, O. Ouari, M. Schwarzwälder, 1263 A. J. Rossini, F. Aussenac, M. Yulikov, G. Jeschke, 1264
C. Copéret, A. Lesage, P. Tordo, and L. Emsley, J. Am. Chem. Soc. 135, 12790 (2013).

[24] H. Takahashi, D. Lee, L. Dubois, M. Bardet, S. Hediger, and G. De Paëpe, Angew. Chemie - Int. Ed. 51, 11766 (2012).

[25] H. Takahashi, I. Ayala, M. Bardet, G. De Paëpe, J. P. Simorre, and S. Hediger, J. Am. Chem. Soc. 135, 5105 (2013).

[26] H. Takahashi, C. Fernández-de Alba, D. Lee, V. Maurel, S. Gambarelli, M. Bardet, S. Hediger, A.-L. Barra, and G. De Paëpe, J. Magn. Reson. 239, 91 (2014).

[27] H. Takahashi, S. Hediger, and G. De Paëpe, Chem. Commun. 49, 9479 (2013).

[28] D. Le, G. Casano, T. N. T. Phan, F. Ziarelli, O. Ouari, F. Aussenac, P. Thureau, G. Mollica, D. Gigmes, P. Tordo, and S. Viel, Macromolecules 47, 3909 (2014).

[29] C. Fernández-de Alba, H. Takahashi, A. Richard, Y. Chenavier, L. Dubois, V. Maurel, D. Lee, S. Hediger, and G. De Paëpe, Chem. - A Eur. J. 21, 4512 (2015).

[30] G. Mathies, M. A. Caporini, V. K. Michaelis, Y. Liu, K.-N. N. Hu, D. Mance, J. L. Zweier, M. Rosay, M. Baldus, and R. G. Griffin, Angew. Chemie 127, 11936 (2015).

[31] C. Sauvee, G. Casano, S. Abel, A. Rockenbauer, D. Akhmetzyanov, H. Karoui, D. Siri, F. Aussenac, W. Maas, R. T. Weber, T. Prisner, M. Rosay, P. Tordo, and O. Ouari, Chem. - A Eur. J. 22, 5598 (2016).

[32] A. N. Smith, U. T. Twahir, T. Dubroca, G. E. Fanucci, and J. R. Long, J. Phys. Chem. B 120, 7880 (2016).

[33] A. P. Jagtap, M.-A. Geiger, D. Stöppler, M. OrwickRydmark, H. Oschkinat, and S. T. Sigurdsson, Chem. Commun. 52, 7020 (2016).

[34] K. R. Thurber, A. Potapov, W.-M. Yau, and R. Tycko, J. Magn. Reson. 226, 100 (2012).

[35] Y. Matsuki, K. Ueda, T. Idehara, R. Ikeda, I. Ogawa, S. Nakamura, M. Toda, J. P. Amoureux, and T. Fujiwara, J. Magn. Reson. 225, 1 (2012).

[36] E. Bouleau, P. Saint-Bonnet, F. Mentink-Vigier, H. Takahashi, J.-F. Jacquot, M. Bardet, F. Aussenac, A. Purea, F. Engelke, S. Hediger, D. Lee, and G. De Paëpe, Chem. Sci. 6, 6806 (2015).

[37] D. Lee, E. Bouleau, P. Saint-Bonnet, S. Hediger, and G. De Paëpe, J. Magn. Reson. 264, 116 (2016).

[38] K. R. Thurber and R. Tycko, J. Magn. Reson. 264, 99 (2016).

[39] Y. Matsuki, T. Idehara, J. Fukazawa, and T. Fujiwara, J. Magn. Reson. 264, 107 (2016).

[40] T. F. Kemp, H. R. Dannatt, N. S. Barrow, A. Watts, S. P. Brown, M. E. Newton, and R. Dupree, J. Magn. Reson. 265, 77 (2016).

[41] S. Chaudhari, P. Berruyer, D. Gajan, C. Reiter, F. Engelke, D. Silverio, C. Copéret, M. Lelli, A. Lesage, and L. Emsley, Phys. Chem. Chem. Phys. 18, 10616 (2016).

[42] M. Rosay, M. Blank, and F. Engelke, J. Magn. Reson. 264, 88 (2016).

[43] S. Lange, A. H. Linden, U. Akbey, W. Trent Franks, N. M. Loening, B.-J. van Rossum, and H. Oschkinat, J. Magn. Reson. , 13 (2012).

[44] Ü. Akbey, A. H. Linden, and H. Oschkinat, Appl. Magn. Reson. 43, 81 (2012). 
[45] M.-A. Geiger, M. Orwick-Rydmark, K. Märker, W. T. ${ }_{1306}$ Franks, D. Akhmetzyanov, D. Stöppler, M. Zinke, 1307 E. Specker, M. Nazaré, A. Diehl, B.-J. van Rossum, 1308 F. Aussenac, T. Prisner, Ü. Akbey, and H. Oschki- 1309 nat, Phys. Chem. Chem. Phys. 18, 30696 (2016). 1310

[46] A. J. Rossini, A. Zagdoun, M. Lelli, D. Gajan, 1311 F. Rascón, M. Rosay, W. E. Maas, C. Coperet, 1312 A. Lesage, and L. Emsley, Chem. Sci. 3, 108 (2012). 1313

[47] D. Kubicki, G. Casano, M. Schwarzwalder, S. Abel, 1314 C. Sauvee, K. Genevan, M. Yulikov, A. J. Rossini, 1315 G. Jeschke, C. Coperet, A. Lesage, P. Tordo, O. Ouari, 1316 and L. Emsley, Chem. Sci. , 1 (2015).

[48] F. A. Perras, R. R. Reinig, I. I. Slowing, A. D. Sadow, ${ }_{1318}$ and M. Pruski, Phys. Chem. Chem. Phys. 18, 651319 (2015).

[49] B. Corzilius, L. B. Andreas, A. a. Smith, Q. Z. Ni, and ${ }_{1321}$ R. G. Griffin, J. Magn. Reson. 240, 113 (2014). 1322

[50] F. Mentink-Vigier, S. Paul, D. Lee, A. Feintuch, 1323 S. Hediger, S. Vega, and G. De Paëpe, Phys. Chem. 1324 Chem. Phys. 17, 21824 (2015). 1325

[51] K. R. Thurber and R. Tycko, J. Chem. Phys. 137, 1326 084508 (2012).

[52] F. Mentink-Vigier, U. Akbey, Y. Hovav, S. Vega, 1328 H. Oschkinat, and A. Feintuch, J. Magn. Reson. 224, 1329 13 (2012)

[53] K. R. Thurber and R. Tycko, J. Chem. Phys. 140, 1331 184201 (2014).

[54] F. Mentink-Vigier, U. Akbey, H. Oschkinat, S. Vega, 1333 and A. Feintuch, J. Magn. Reson. 258, 102 (2015). 1334

[55] D. Mance, P. Gast, M. Huber, M. Baldus, and K. L. 1335 Ivanov, J. Chem. Phys. 142, 234201 (2015).

[56] A. Karabanov, I. Kuprov, G. T. P. Charnock, 1337 A. van der Drift, L. J. Edwards, and W. Kocken- 1338 berger, J. Chem. Phys. 135, 084106 (2011).

[57] A. Karabanov, A. van der Drift, L. J. Edwards, 1340 I. Kuprov, and W. Kockenberger, PCCP Phys. Chem. 1341 Chem. Phys. 14, 2658 (2012).

[58] C. Zener, Proc. R. Soc. A Math. Phys. Eng. Sci. 137, 1343 696 (1932).

[59] T. Davis, http://faculty.cse.tamu.edu/davis /suites- $\mathbf{1 3 4 5}$ parse.html.
[60] M. Gafurov, S. Lyubenova, V. Denysenkov, O. Ouari, H. Karoui, F. Le Moigne, P. Tordo, and T. F. Prisner, Appl. Magn. Reson. 37, 505 (2010).

[61] K.-N. N. Hu, C. Song, H.-H. Yu, T. M. Swager, and R. G. Griffin, J. Chem. Phys. 128, 052302 (2008).

[62] M. H. Levitt and L. D. Bari, Bull. Magn. Reson. 16, 94.

[63] T. Levante and R. Ernst, Chem. Phys. Lett. 241, 73 (1995).

[64] N. V. Vitanov, Phys. Rev. A 59, 988 (1999).

[65] O. Leifson and C. D. Jeffries, Phys. Rev. 122, 1781 (1961).

[66] M. Ernst and B. H. Meier, in Stud. Phys. Theo. Chem., Vol. 84, edited by A. I. and T. Asakura (Elsevier Science B.V, 1998) Chap. 4, pp. 83-121.

[67] N. Bloembergen, Physica 15, 386 (1949).

[68] G. R. Khutsishvili, Sov. Phys. JETP 42, 909 (1962).

[69] M. Goldman, Phys. Rev. 138, A1675 (1965).

[70] G. R. Khutsishvili, Sov. Phys. Uspekhi 11, 802 (1969).

[71] J. P. Wolfe, Phys. Rev. Lett. 31, 907 (1973).

[72] D. Suter and R. Ernst, Phys. Rev. B 32, 5608 (1985).

[73] a. Karabanov, D. Wiśniewski, I. Lesanovsky, and W. Köckenberger, Phys. Rev. Lett. 115, 020404 (2015).

[74] K.-N. N. Hu, G. T. Debelouchina, A. A. Smith, and R. G. Griffin, J. Chem. Phys. 134, 125105 (2011).

[75] Y. Hovav, A. Feintuch, and S. Vega, J. Chem. Phys. 134, 074509 (2011).

[76] Y. Hovav, A. Feintuch, and S. Vega, J. Magn. Reson. 214, 29 (2012).

[77] O. Lafon, M. Rosay, F. Aussenac, X. Lu, J. Trébosc, O. Cristini, C. Kinowski, N. Touati, H. Vezin, and J. P. Amoureux, Angew. Chemie - Int. Ed. 50, 8367 (2011).

[78] O. Lafon, A. S. L. Thankamony, M. Rosay, F. Aussenac, X. Lu, J. Trébosc, V. Bout-Roumazeilles, H. Vezin, and J. P. Amoureux, Chem. Comm. 49, 2864 (2013).

[79] A. J. Rossini, A. Zagdoun, F. Hegner, M. Schwarzwälder, D. Gajan, C. Copéret, A. Lesage, and L. Emsley, J. Am. Chem. Soc. 134, 16899 (2012). 